\title{
The first record of Late Jurassic megateuthidid belemnites - Chuvashiteuthis aenigmatica gen. et sp. nov. from the upper Kimmeridgian of Central Russia
}

\author{
Alexel P. Ippolitov, Alexander Yu. Berezin, Mikhall A. RogOV \& BhaWANisingh G. Desal
}

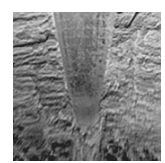

\begin{abstract}
Present paper discusses finds of peculiar Upper Kimmeridgian belemnitids from Central Russia. Systematic morphological investigation, combined with biometrical comparison and mineralogical study, has shown that these belemnitids should be classified within the family Megateuthididae. They are described herein as Chuvashiteuthis aenigmatica gen. et sp. nov. These finds are the youngest record of megateuthidid belemnites, which are considered to die out during the Bathonian, thus extending the total range of the family by 12 Ma. The described species co-occurs with scarce and suppressed Boreal belemnites and abundant ammonites of Boreal-Atlantic and Tethyan affinities, supposing similar origin also for the newly described genus. In addition to new data on belemnites, stratigraphical distribution of remarkable "Laevaptychus-bearing" horizons in the Kimmeridgian and Volgian of the Russian Platform is briefly outlined. • Key words: Belemnites, Megateuthididae, Chuvashiteuthis aenigmatica gen. et sp. nov., rostrum, phragmocone, biogeography.
\end{abstract}

IPPOlitov, A.P., Berezin, YU., Rogov, M.A. \& DeSAI, B.G. 2017. The first record of Late Jurassic megateuthidid belemnites - Chuvashiteuthis aenigmatica gen. et sp. nov. from the upper Kimmeridgian of Central Russia. Bulletin of Geosciences 92(3), 357-372 (6 figures, 1 table), Czech Geological Survey, Prague, ISSN 1214-1119. Manuscript received November 26, 2016; accepted in revised form July 7, 2017; published online September 30, 2017; issued September 30, 2017.

Alexei P. Ippolitov \& Mikhail A. Rogov, Geological Institute of Russian Academy of Sciences, Pyzhevski Lane 7, 119017, Moscow, Russia; ippolitov.ap@gmail.com, russianjurassic@gmail.com•Alexander Yu. Berezin, «Terra Incognita» Chuvash Society of Archaeology and Natural History, Michman Pavlov street 13-2, ZIP code: 428034 Cheboksary, Russia; terra3@inbox.ru - Bhawanisingh G. Desai, Pandit Deendayal Petroleum University, Gandhinagar District, Raisan, 382007 Gujarat, India; bhawanigd@gmail.com

Upper Jurassic belemnite assemblages of Central Russia are represented by two families - abundant and highly diversified Cylindroteuthididae of the Boreal origin and relatively rare Belemnopseidae (a single genus Hibolithes de Montfort, 1808) of the Tethyan origin. The Boreal family is a dominating group throughout the Callovian-Upper Jurassic sequence of the region, while Tethyan Hibolithes occurs at certain succession intervals and sometimes at isolated invasion levels. They are represented by small-sized rostra and are probably suppressed (Gustomesov 1961). Additionally, members of the order Belemnotheutida, having thin sheath-like rostra can also sometimes occur (see Rogov \& Bizikov 2008).

During the field seasons 2008-2009, two unusual short and conical belemnoid rostra with dorsal keel were discovered in the Upper Kimmeridgian deposits of Chuvashia region. These forms differ morphologically from any group of belemnites previously known from the Upper Jurassic of Russian Platform.
The present paper deals with this problematic material, discussing its taxonomic assignment and possible phylogenetic relationships. Rostra are described herein as Chuvashiteuthis aenigmatica gen. et sp. nov., and related discussion considering the development of belemnite faunas of Russian Platform during the Late Jurassic, is also provided below.

\section{Geological setting}

The first specimen of Chuvashiteuthis aenigmatica gen. et sp. nov. was found in the locality Sovhoznyi, $60 \mathrm{~km}$ to the west from the Cheboksary City $\left(56.033931^{\circ} \mathrm{N}\right.$, $46.284184^{\circ}$ E; Fig. 1B), and looking like isolated Kimmeridgian outcrop among forested slope of the Sura river bank. Exhaustive targeted search was undertaken by the authors next year in numerous localities of the similar age 


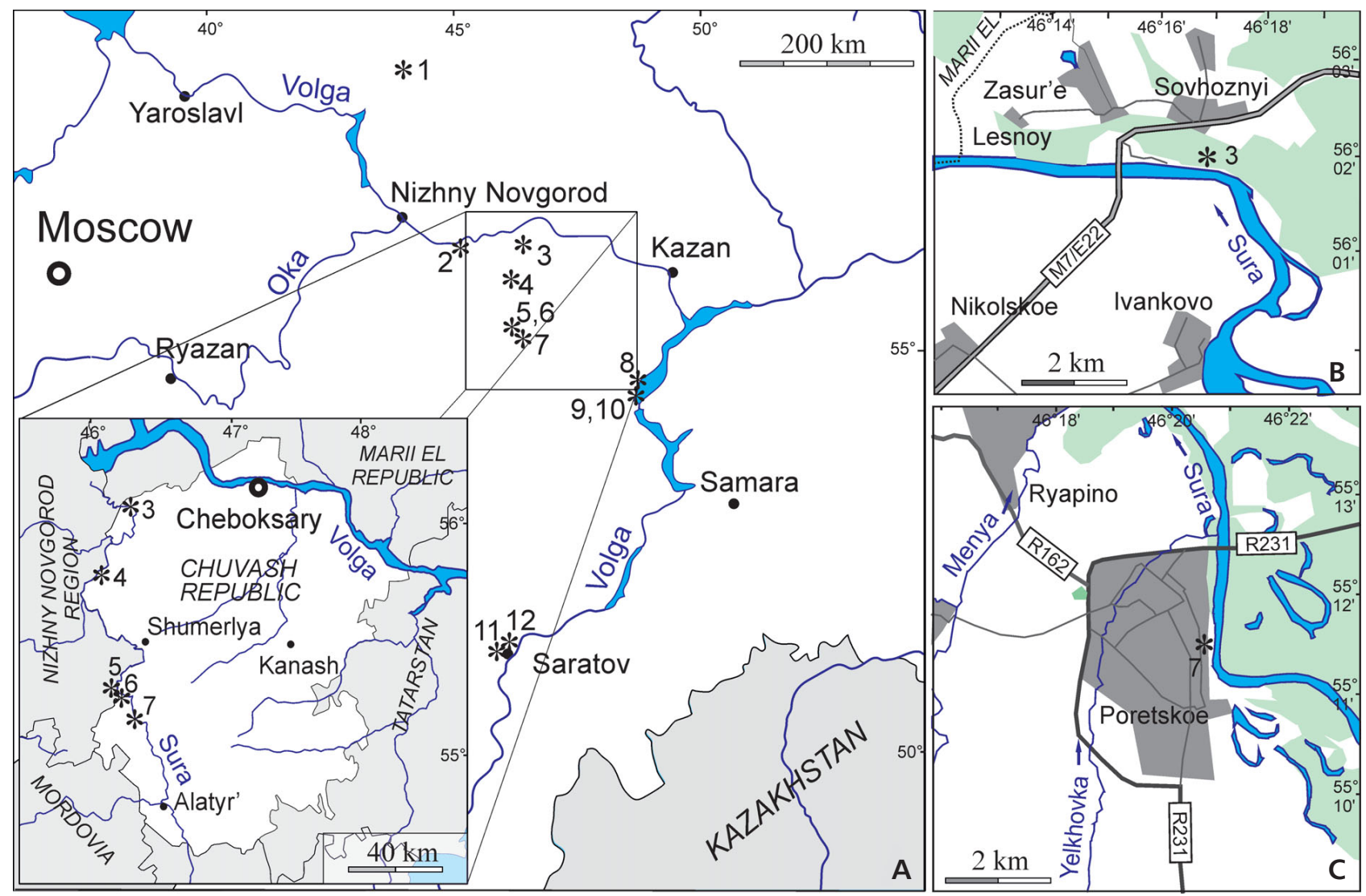

Figure 1. Location of the sections mentioned in the paper (A), including details of those containing Chuvashiteuthis gen. nov. finds (B, C). Localities: 1 - Mikhalenino; 2 - Prosek; 3 - Sovhoznyi; 4 - Khvadukassy; 5 - Murzicy; 6 - Kozlovka; 7 - Poretskoe; 8 - Tarkhanovskaya Pristan'; 9 - Undory; 10 - Gorodischi; 11 - Sokur Quarry (Saratov City); 12 - Dubki.

within the region. And as a result, one more specimen was found on the bank of the river Sura near Poretskoe Village $\left(55.192631^{\circ} \mathrm{N}, 46.342519^{\circ} \mathrm{E}\right.$; Fig. 1C), approximately $95 \mathrm{~km}$ to the south from the first locality.

Both rostra mentioned above come from the talus, but for both finds the age can be determined very precisely. Hypsometrical positions of finds show that in both cases rostra came from thick clayish Novikovo Formation of the late Kimmeridgian-early Volgian age. More detailed information is provided by co-occurring faunal remains. Kimmeridgian succession of the region does not contain abundant macrofauna, but in both cases our finds were accompanied by evident "faunal spots" in the scree, containing numerous small belemnite rostra of family Cylindroteuthididae, Ostrea-like bivalve shells, echinoderm spines, rare serpulid tubes, small gastropods and very numerous laevaptychi (Fig. 2), originating from the certain stratigraphic level (called below "the Laevaptychus horizon" or "the Laevaptychus-bearing horizon"). This complex was discovered in two more sections over the region: Khvadukassy and Kozlovka, however, no Chuvashiteuthis aenigmatica gen. et sp. nov. were found there.

Numerous belemnite rostra in the complex accompanying our finds represent the Boreal family Cylindroteuthididae, and belong to a single species Boreioteuthis troslayana (d'Orbigny, 1850) which indicates Kimmeridgian to middle Volgian age (Dzyuba 2004). All the specimens, counting totally several dozens of fragments, represented only by small rostra, usually not exceeding $5 \mathrm{~mm}$ in diameter and a single specimen reaching $9 \mathrm{~mm}$ (Fig. 2M).

Among poorly preserved ammonite remains, found in Sovhoznyi in association with one of the strange rostra described below, the following species were determined: Aulacostephanus cf. volgensis (Vischn.), Sarmatisphinctes cf. subborealis (Kutek et Zeiss), and numerous aptychi Laevaptychus sp. ind. This assemblage is characteristic for the lower part of the autissiodorensis Zone of the upper Kimmeridgian. Taking into account the presence of aspidoceratid ammonites and/or laevaptychi in the lowermost horizon of the autissiodorensis Zone only ["aff. rebholzi" horizon, see Rogov 2010; here and throughout the text the term "horizon"/"biohorizon" is used according to the practice of ammonite biostratigraphy, for indicating the smallest biostratigraphically discernible intervals, see Page (1995) and Rogov et al. (2012) for further details], studied assemblage seems to belong to the basal part of the 

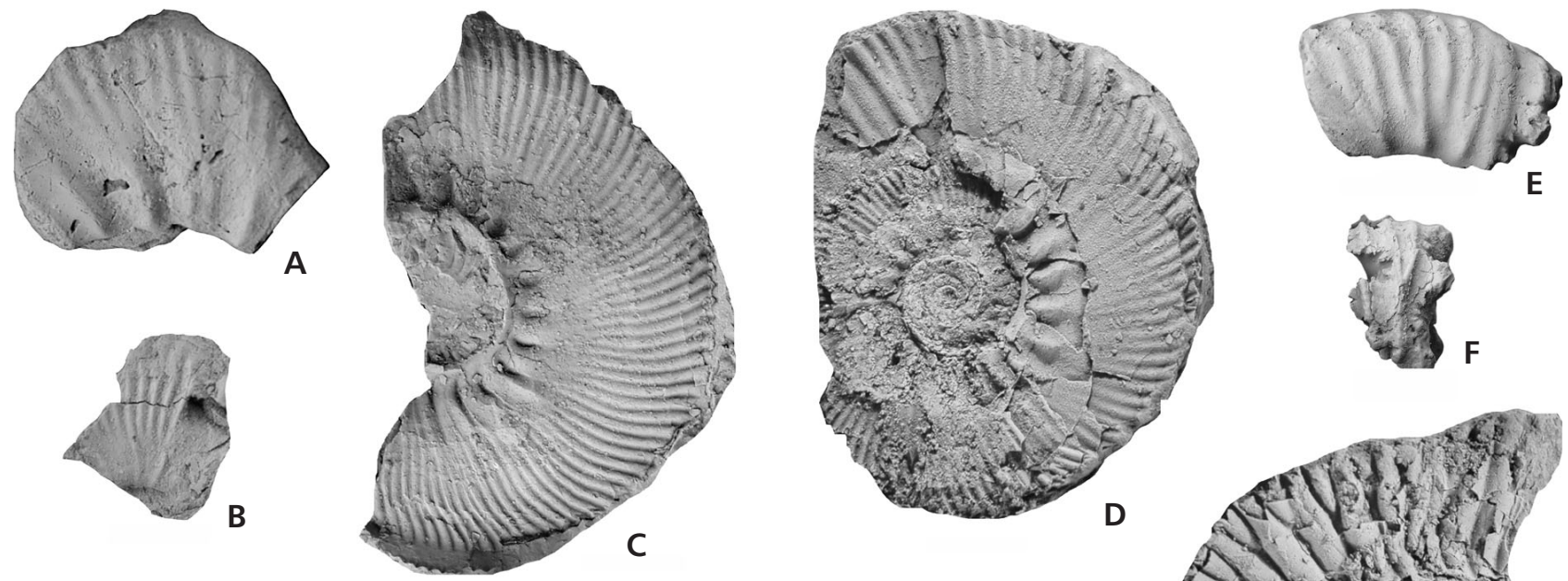

(⿻)
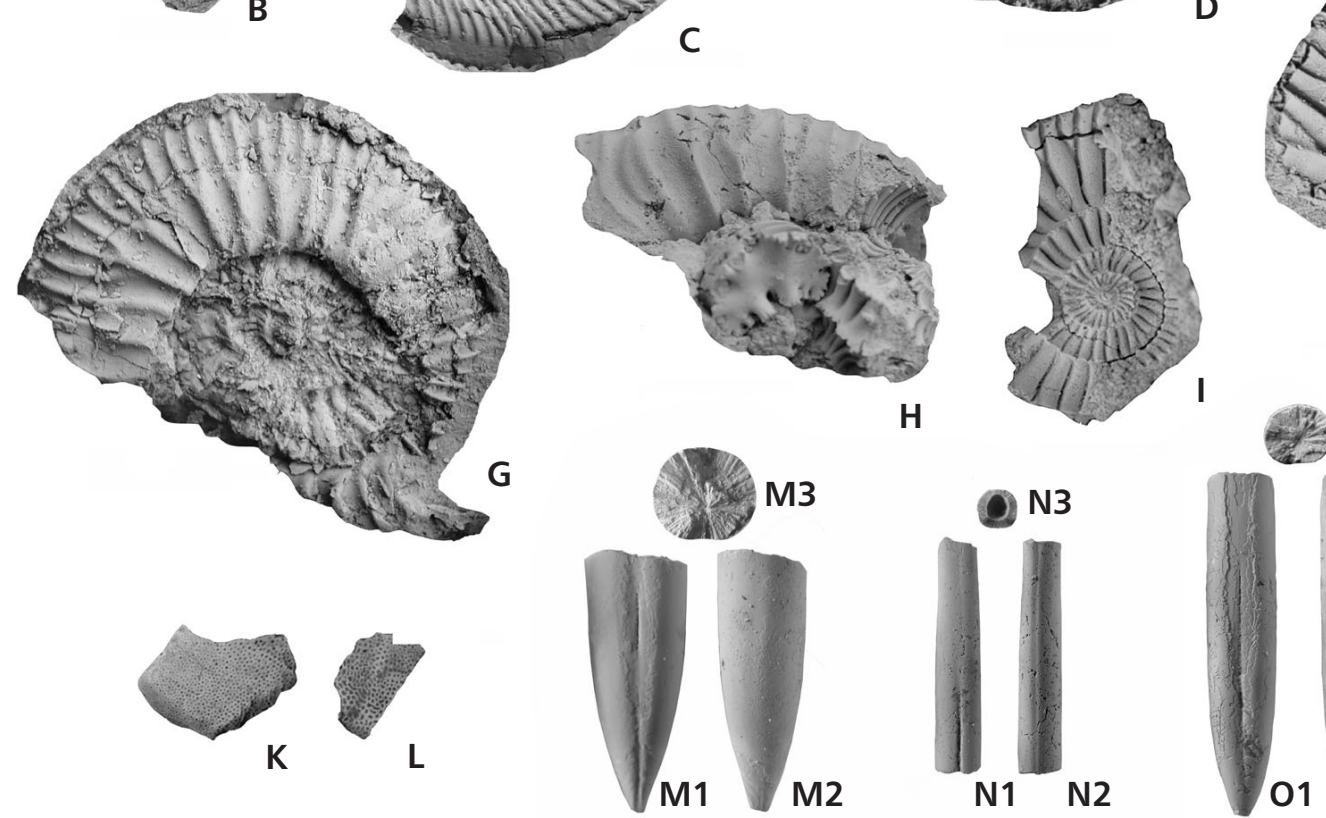

H
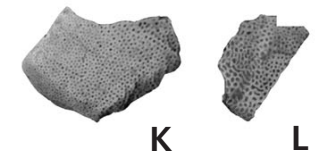

L
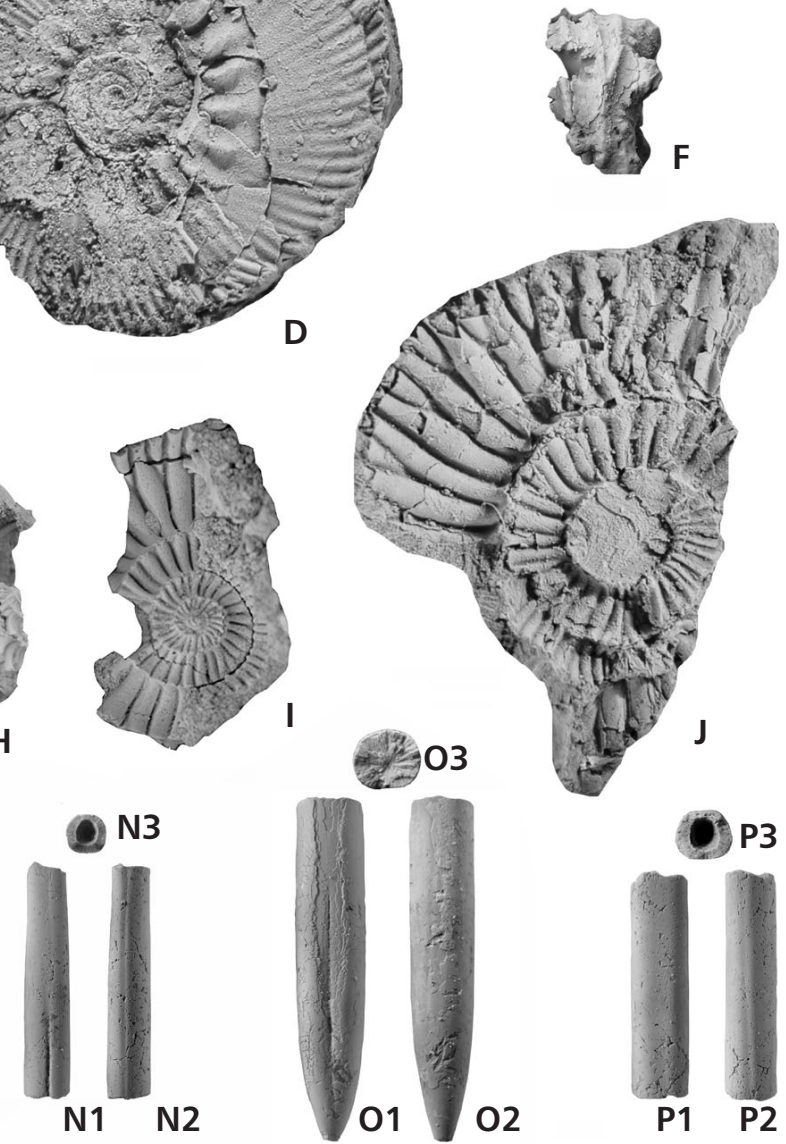

P3

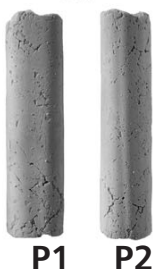

Figure 2. Cephalopod complex of the upper Kimmeridgian autissiodorensis Zone, co-occurring in "faunal spots" together with Chuvashiteuthis gen. nov. (all figures natural size). A, B - Aulacostephanus cf. volgensis (Vischniakoff, 1875); A - VSGM BP-11463, Sovhoznyi; B - VSGM BP-11464, Sovhoznyi. • C, D - Aulacostephanus volgensis (Vischniakoff, 1875); C - VSGM BP-11462, Gorodischi; D - Murzicy, subborealis horizon, specimen lost. $•$ E-Aulacostephanus cf. kirghisensis (d'Orbigny, 1845), VSGM BP-11465, Sovhoznyi. • F, G, H, I-Sarmatisphinctes subborealis (Kutek et Zeiss, 1997); F - VSGM BP-11466, Sovhoznyi; G - VSGM BP-11460, Murzicy, subborealis horizon; J - VSGM BP-11461, Murzicy, subborealis horizon; H-I - Prosek, volgae horizon, both specimens lost. $\bullet \mathrm{K}, \mathrm{L}$ - Laevaptychus sp., Zasur'e, specimens lost. $\bullet \mathrm{M}-\mathrm{P}$ - Boreioteuthis troslayana (d'Orbigny, 1850), juvenile specimens; M - VSGM BP-11440, Kozlovka, apical part of the largest available specimen (M1 - ventral view; M2 - left lateral view; M3 - cross-section at the anterior end); N - VSGM BP-11441, Sovhoznyi, specimen with untypically incised furrow on wide ventral flattening (N1 - ventral view; N2 - left lateral view; N3 - anterior end); O - VSGM BP-11442, Kozlovka (O1 - ventral view; O2 - left lateral view; O3 - cross-section at the anterior end, nearby protoconch); P - VSGM BP-11443, Kozlovka, specimen with strong ventral flattening (P1 - ventral view; P2 - left lateral view; P3 - anterior end).

autissiodorensis Zone. It should be noted, however, that ammonites are represented by poor, partially preserved moulds only and possibility of slightly older age of the discussed assemblage cannot be excluded.

Laevaptychus-bearing aspidoceratid ammonites are not typical for the uppermost Jurassic of the Russian Platform, and up to date only 5 intervals rich in such aptychi are known across the Kimmeridgian-lower Volgian (Fig. 3). Lowermost level A, belonging to the bayi horizon of the lower Kimmeridgian, is known from the Kostroma region only (Głowniak et al. 2010). Level B, characterized by both aspidoceratid ammonites and laevaptychi expands over the upper Kimmeridgian mutabilis Zone, with exception of its presence in lowermost part (Rogov et al. 2017). Most well-recognizable level $\mathrm{C}$ is also characterized by numerous occurrences of Aspidoceras spp., and is spanning across the upper part of the eudoxus Zone. It includes yo horizon and the level above, tentatively named robertianum horizon, which is nearly lacking aulacostephanid ammonites (a single specimen of 
Aulacostephanus sp. is known from this unit). However, it is characterized by numerous occurrences of Aspidoceras quercynum (Hantzp.), Sutneria ex gr. eumela (d'Orb.), Tolvericeras sevogodense (Contini et Hantzp.), T. robertianum (Enay, Gallois et Etches), Discosphinctoides sp. as well as relatively rare Hoplocardioceras cf. elegans (Spath), and Neochetoceras cf. acallopistum (Font.). The latter assemblage is known mainly from the Middle Volga area, including Chuvashia. In the reference section Khvadukassy $\left(55.769722^{\circ} \mathrm{N}, 46.094167^{\circ} \mathrm{E}\right.$, Fig. 1) it occurs within the 3.5 -m-thick clayey member with thin $(0.3 \mathrm{~m})$ limestone interband located $0.5 \mathrm{~m}$ below the top. Ammonites A. quercynum and Discosphinctoides are the most numerous here (Fig. 3). Above the aforementioned strata two thinner Laevaptychus-bearing intervals are known: level D in the base of the autissiodorensis Zone (aff. rebholzi horizon, see Rogov 2010) and level E in the lowermost part of the lower Volgian puschi Zone (neoburgense horizon, see Rogov 2004).

In our opinion, even considering that no ammonites were found in Poretskoe, both finds of Chuvashiteuthis aenigmatica gen. et sp. nov. originate from the aptychus-bearing horizon D and therefore are late Kimmeridgian (earliest autissiodorensis Chron) in age.

\section{Methods of study}

Mineralogical composition of rostra was achieved using $\mathrm{X}$-ray diffraction analysis on DRON-3M machine in the laboratory of Geological Faculty of Moscow State University.

All measurements of rostra were performed according to scheme, widely accepted in Russian literature for Boreal belemnites (see Dzyuba 2004), with abbreviation translated to English by Dzyuba (2012, fig. 2). Abbreviations are as following: $\mathrm{R}$ - total preserved length; DV - dorsoventral diameter near the tip of the alveolus; LL - lateral diameter near the tip of the alveolus; PA - length of the postalveolar part of the rostrum ("rostrum solidum"); $\mathrm{dv}$ - dorsoventral diameter in the apical region of the rostrum; 11 - lateral diameter in the apical region of the rostrum; $\alpha$ - apical angle of the rostrum.

Among these measurements DV is traditionally accepted as $100 \%$, relatively to which all other linear parameters calculated also as \%, providing principal ratios describing the shape of the rostrum. The 11 and $\mathrm{dv}$ were not measured in our material, as in perfectly conical rostra the demarcation between stem and apical regions is not possible.

Phragmocones are usually neglected in systematic belemnite studies. They are considered to be a conservative element of belemnite skeleton (Jeletzky 1966), thus being not helpful in routine systematic work. However, in some cases, phragmocone characters may be treated as apomorphies defining ordinal-rank taxa (e.g. Diplobelida, see Jeletzky 1981). Nevertheless, even reviews dealing with larger taxa often lack their measurements, discussing characters as qualitative (e.g. Doyle \& Shakides 2004).

Phragmocones were measured according to our new scheme, presented on Figure 4, either on free phragmocones or by the imprints of their elements preserved within alveola. Measurements of phragmocones were obtained using digital caliper and/or measuring ocular of the microscope. These dimensions include:

$\beta(\mathrm{dv})$ - phragmocone angle in profile; $\beta(11)$ - phragmocone angle in outline [in case when juvenile part of phragmocone was not preserved, for obtaining phragmocone angles we used extrapolated position of its tip, produced by intersection of two straight lines connecting centres of LAFC with centres of first available full camera (Fig. 4)]; ch - camera height, dorsoventral diameter of phragmocone measured in the last available fully preserved camera (LAFC); cw - camera width; lateral diameter of phragmocone, measured in the LAFC; $\mathrm{cl}$ - camera length, measured in the LAFC; pw - proostracum width, measured in the LAFC. Among measurements of phragmocone, ch was accepted as $100 \%$, but proostracum width was calculated as $\%$ from $\mathrm{cw}$.

To compare our material with different coleoids and most potentially related belemnite taxa, we used our own collections and examined specimens from numerous previously published collections, the most important of which are originals by Sachs \& Nalnjaeva (1975), kept in the Central Siberian Geological Museum (Novosibirsk), by Gustomesov (1960a) and by Barskov (in Mitta et al. 2004), both kept in the Vernadsky State Geological Museum (VSGM, Moscow). Together these collections cover the total biodiversity of megateuthidid genera previously known for the Middle Jurassic of Russia and possibly related to Chuvashiteuthis aenigmatica gen. et sp. nov.

Institutional abbreviations. - VSGM - Vernadsky State Geological Museum, Moscow.

\section{Systematic palaeontology}

This published work and the nomenclatural acts it contains have been registered in Zoobank: http://zoobank.org/urn:lsid:zoobank.org:pub:OBD4BD5D -F13C-4AF2-B088-6B0942203E16.

Subclass Coleoidea Bather, 1888

Order Belemnitida von Zittel, 1895

Suborder Belemnitina von Zittel, 1895

Family Megateuthididae Sachs et Nalnjaeva, 1967

Remarks. - In the present paper we follow "wide" concept 


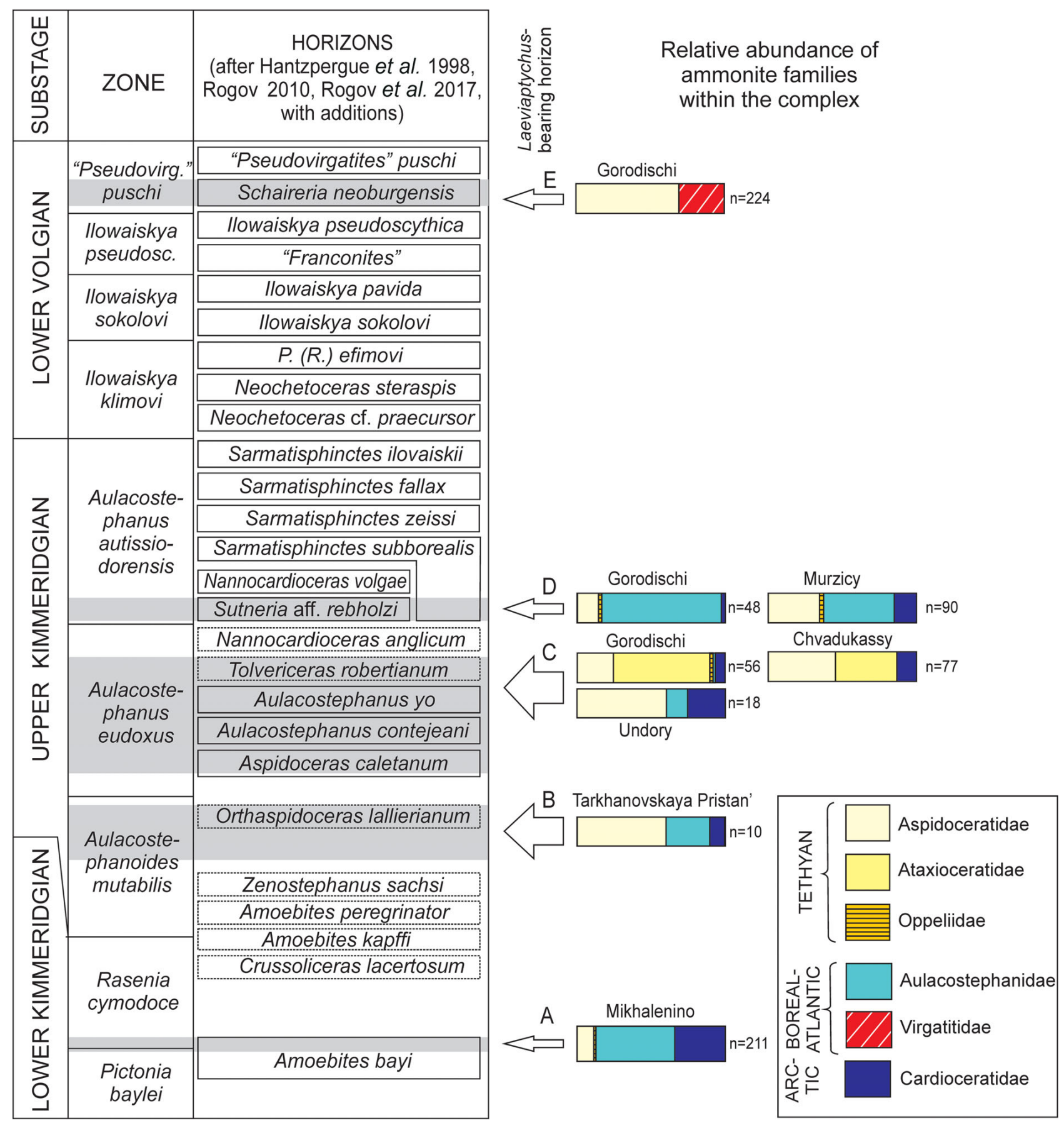

Figure 3. Positions of Laevapthychus-bearing horizons in the Kimmeridgian-lower Volgian succession of the Middle Volga area.

of Megateuthididae closely to as it was defined in the recent revision by Dzyuba et al. (2015). This concept is shared by the majority of recent authors (Riegraf 1995 as "Acrocoelitidae", Riegraf et al. 1998, Dzyuba \& Weis 2015, Dzyuba et al. 2015). It places into the family Megateuthididae numerous genera, characterized by very different rostrum shapes and furrowing patterns, often with epirostra and normally of markedly conical shape at the earliest growth stages. The latter two are the most typical attributes of the family, however, no one single character from the diagnosis can be considered as synapomorphy strictly defining the family and clearly separating it from more ancient family Passaloteuthididae Naef, 1922, and thus polyphyletic nature of Megateuthididae is not excluded. 


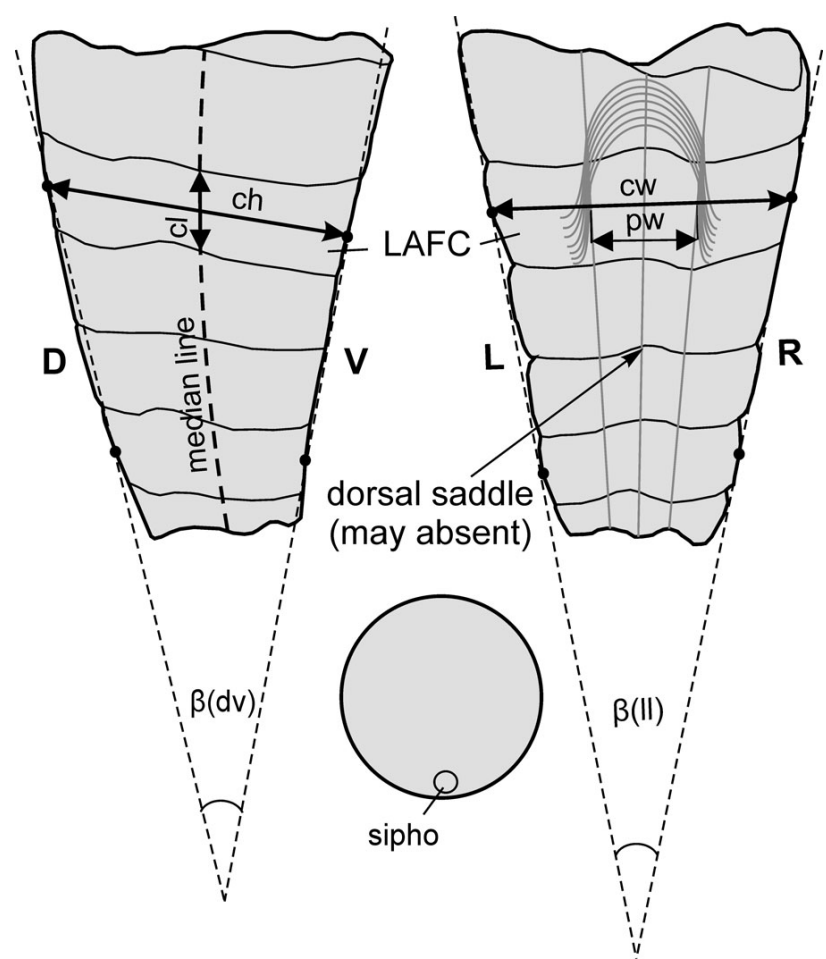

Figure 4. Phragmocone dimensions and their abbreviations: V - ventral side; $\mathrm{D}$ - dorsal side; $\mathrm{L}$ - left side; $\mathrm{R}$ - right side; $\mathrm{LAFC} \mathrm{-} \mathrm{latest} \mathrm{available}$ fully preserved camera.

The alternative "narrow" concept offered by Schlegelmilch (1998) and followed by few recent authors (Dera et al. 2016), restricts the family only to its type genus plus Mesoteuthis Lissajous, 1915. However, phylogenetic interrelations between most Toarcian-Middle Jurassic genera are predominantly problematic rather than well-established (Schlegelmilch 1998, text-fig. 15). As a result, in frames of "narrow" concept clearly related taxa may become disjuncted and transferred to different families (e.g. Homaloteuthis Stolley, 1919 and Eocylindroteuthis Riegraf, 1980, having very similar ontogeny and both close to Megateuthis by general shape and stratigraphic position - see Weis \& Mariotti 2008), while other taxa, not present in South Germany, are not discussed at all (e.g. Paramegateuthis Gustomesov, 1960a). Considering the aforesaid, further subdivision of Megateuthididae "sensu lato" currently looks premature. The re-assignment of the whole passaloteuthidid-megateuthidid complex into phylogenetic clusters is possible, but should be based on well-established phylogenetic relations, ideally traced within the successions, rather than existing speculative hypotheses.

\section{Genus Chuvashiteuthis Ippolitov et Berezin gen. nov.}

Type species. - Chuvashiteuthis aenigmatica Ippolitov et Berezin sp. nov.
Etymology. - After the name of administrative region (Chuvash Republic, or Chuvashia) in Central Russia, where the type species originates from.

Diagnosis. - Conical rostrum with narrow dorsal ridge, starting from the apex and extending up to the alveolar part of the rostrum.

Remarks. - From all known megateuthidid genera Chuvashiteuthis gen. nov. differs by the presence of dorsal keel on the rostrum, running from the apex throughout the whole length of the rostrum, slowly declining anteriorly and formed by modified, closely spaced and subparallel dorsolateral apical furrows. Despite the sipho is not preserved in our material, the dorsal position of the keel becomes evident by the observation that the proostracum is located on the same side.

Morphologically closest genus Paramegateuthis Gustomesov, 1960a usually has widely spaced furrows, which tend to diverge and quickly flatten anteriorly. Also it has slightly larger alveolar angle - from $18-19^{\circ}$ (Dzyuba, personal communication 2017) up to 27-29 (see Sachs \& Nalnjaeva 1975). Homaloteuthis Stolley, 1919, which can possess rostrum shape, similar to Chuvashiteuthis gen. nov., neither has dorsal keel nor incised apical dorso-lateral grooves.

Other species. - Unknown.

Occurrence. - The upper Kimmeridgian of Central Russia.

\section{Chuvashiteuthis aenigmatica Ippolitov et Berezin sp. nov.}

Figure 5A, B

Holotype. - VSGM BP-11438, complete rostrum with an imprint of proostracum inside the alveolus; Sovhozhyi; upper Kimmeridgian, ?autissiodorensis Zone, aff. rebholzi horizon of Chuvash Republic, Central Russia.

Paratype. - VSGM BP-11439, complete rostrum without traces of phragmocone or proostracum, Poretskoe. Upper Kimmeridgian, ?autissiodorensis Zone, aff. rebholzi horizon of Chuvash Republic, Central Russia.

Type horizon and locality. - The upper Kimmeridgian, ?autissiodorensis Zone, aff. rebholzi horizon; Sovhoznyi (Chuvash Republic, European Russia).

Material. - Holotype and paratype only.

Etymology. - From the Greek word " $\alpha$ 'iv $\gamma \mu \alpha$ " - a riddle, an enigma, in order to underline uncertain origin of the species. 

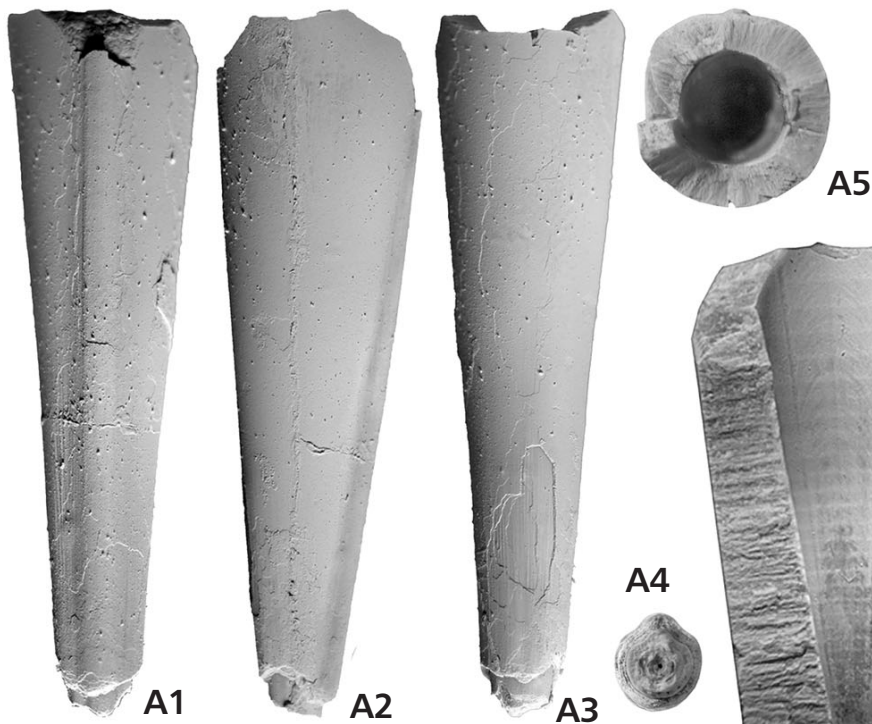

A2
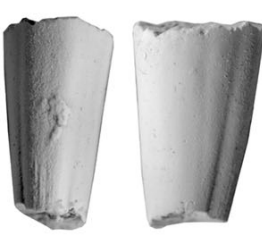

C1

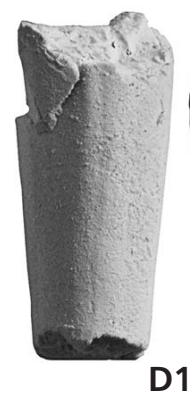

C2
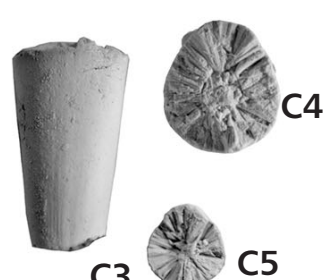

C3
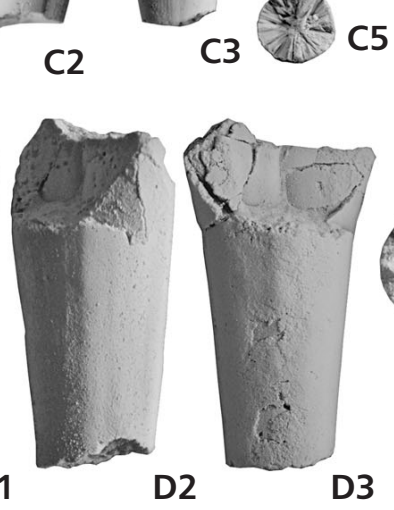

C5

Figure 5. Chuvashiteuthis aenigmatica gen. et sp. nov. from the upper Kimmeridgian of Chuvashia (A, B) and stratigraphically closest megateuthidid belemnites from India (C, D) and north of European Russia (E, F). A, B - Chuvashiteuthis aenigmatica gen. et sp. nov.; A - holotype VSGM BP-11438, Sovhoznyi (A1 - dorsal view; A2 - left lateral view; A3 - ventral view; A4 - cross-section at the apex; A5 - cross-section at the anterior end; A6 - dorsal side of the alveolus, showing imprint of the proostracum); B - paratype VSGM BP-11439, Poretskoe (B1 - dorsal view; B2 - right lateral view; B3 - ventral view; B4 - cross-section at the anterior end); both (A, B) are from upper Kimmeridgian, ?autissiodorensis Zone, aff. rebholzi horizon. - C, D - Megateuthididae gen. et sp. nov.; C - VSGM BP-11458 (C1 - dorsal view; C2 - left lateral view; C3 - ventral view; C4 - cross-section at the anterior end of a fragment; C5 - cross-section at the posterior end); D - VSGM BP-11459 (D1 - dorsal view; D2 - left lateral view; D3 - ventral view; D4 - cross-section at the anterior end of a fragment); both (C, D) are from the middle Bathonian of Kachchh, India. $\bullet$ E - Paramegateuthis timanensis (Gustomesov, 1960a), holotype VSGM VI-126/256, Komi Rep., Izhma river, Razlivnoi rapid, lower-?middle Bathonian (E1 - dorsal view; E2 - right lateral view; E3 - ventral view; E4 - cross-section at the anterior end). F - Paramegateuthis ishmensis (Gustomesov, 1960a), holotype VSGM VI-126/254, Komi Rep., Izhma river, Razlivnoi rapid, lower-?middle Bathonian (F1 - dorsal view; F2 - left lateral view; F3 - ventral view; F4 - cross-section at the anterior end). Scale bar is $10 \mathrm{~mm}$ for A1-A5, B-D; $3 \mathrm{~mm}$ for A6 and $5 \mathrm{~mm}$ for E and F.

Diagnosis. - Conical small-sized short rostrum. Dorsolateral apical furrows are closely-spaced, making appearance of a single dorsal narrow ridge lying between them, and run throughout the whole length of the rostrum.

Description. - Rostrum: Small-sized, slightly elongated calcitic rostra, conical both in profile and outline. Apical angle in outline is 8 and $9^{\circ}$, in profile 11.5 and $12^{\circ}$, for holotype and paratype respectively. The cross-section is compressed in the post-alveolar region, slowly becoming circular to the anterior margin of the alveolar part. Margins seen in outline are straight (in the paratype) to very slightly concave (in the holotype). The profile is almost symmetrical, with straight (in the holotype) to very slightly convex 
Table 1. Biometric comparison of Chuvashiteuthis aenigmatica gen. et sp. nov. with some belemnites, belemnotheutids and other morphologically similar taxa. Abbreviations: * - not the latest camera; ** - at posterior cross-section; alveolar tip not preserved.

\begin{tabular}{|c|c|c|c|c|c|c|c|c|c|c|c|c|}
\hline \multirow[t]{2}{*}{ Taxon name } & \multirow{2}{*}{$\begin{array}{l}\text { Specimen No./ } \\
\text { reference to figured } \\
\text { material }\end{array}$} & \multirow{2}{*}{$\begin{array}{l}\text { Locality, region, } \\
\text { age }\end{array}$} & \multicolumn{4}{|c|}{ Rostrum } & \multicolumn{5}{|c|}{ Phragmocone } & \multirow{2}{*}{\begin{tabular}{|c|} 
Proostracum \\
$\mathrm{w}, \mathrm{mm}$ \\
$(\%)$ from $1 \mathrm{l}(\mathrm{p})$
\end{tabular}} \\
\hline & & & $\mathrm{R}, \mathrm{mm}$ & $\begin{array}{c}\mathrm{DV}, \\
\mathrm{mm}(\%)\end{array}$ & $\begin{array}{c}\mathrm{LL}, \\
\mathrm{mm}(\%)\end{array}$ & $\begin{array}{c}\mathrm{PA} \\
\mathrm{mm}(\%)\end{array}$ & $\beta(\mathrm{dv})^{\circ}$ & $\beta(11)^{\circ}$ & $\begin{array}{c}\mathrm{dv}(\mathrm{p}) \\
(\%)\end{array}$ & $\begin{array}{l}11(\mathrm{p}) \\
(\%)\end{array}$ & $\begin{array}{c}\mathrm{cl}, \mathrm{mm} \\
(\%)\end{array}$ & \\
\hline \multicolumn{13}{|c|}{ Chuvashiteuthis gen. nov. } \\
\hline $\begin{array}{l}\text { Chuvashiteuthis } \\
\text { aenigmatica gen. } \\
\text { et. sp.nov. }\end{array}$ & $\begin{array}{l}\text { VSGM BP-11438 } \\
\text { (holotype) }\end{array}$ & $\begin{array}{l}\text { Sovhoznyi; } \\
\text { Chuvashia } \\
\mathrm{J}_{3} \mathrm{~km}_{2}\end{array}$ & 35.0 & $\begin{array}{c}7.4 \\
(100)\end{array}$ & $\begin{array}{l}6.2 \\
(84)\end{array}$ & $\begin{array}{l}21.0 \\
(284)\end{array}$ & 18 & 18 & $\begin{array}{c}5.85 \\
(100)\end{array}$ & $\begin{array}{c}5.85 \\
(100)\end{array}$ & $\begin{array}{c}0.8 \\
(14)\end{array}$ & $\begin{array}{l}3.0 \\
(51)\end{array}$ \\
\hline $\begin{array}{l}\text { Chuvashiteuthis } \\
\text { aenigmatica gen. } \\
\text { et. sp.nov. }\end{array}$ & $\begin{array}{l}\text { VSGM BP-11439 } \\
\text { (paratype) }\end{array}$ & $\begin{array}{l}\text { Poretskoe; } \\
\text { Chuvashia } \\
\mathrm{J}_{3} \mathrm{~km}_{2}\end{array}$ & 28.6 & $\begin{array}{c}7.95 \\
(100)\end{array}$ & $\begin{array}{c}6.4 \\
(81)\end{array}$ & $\begin{array}{l}17.5 \\
(220)\end{array}$ & 18 & 16 & $\begin{array}{l}4.75 \\
(100)\end{array}$ & $\begin{array}{l}4.55 \\
(96)\end{array}$ & - & - \\
\hline \multicolumn{13}{|c|}{ Cylindroteuthidid belemnites } \\
\hline $\begin{array}{l}\text { Pachyteuthis cf. } \\
\text { bodylevskii }\end{array}$ & VSGM BP-11444 & $\begin{array}{l}\text { Sokur; Saratov } \\
\text { region } \mathrm{J}_{2} \mathrm{bt}_{1}\end{array}$ & 56.2 & $\begin{array}{c}19.4 \\
(100)\end{array}$ & $\begin{array}{l}18.5 \\
(95)\end{array}$ & - & 19 & 25 & $\begin{array}{c}8.5 \\
(100)\end{array}$ & $\begin{array}{c}8.2 \\
(96)\end{array}$ & $\begin{array}{c}1.2 \\
(14)\end{array}$ & $\begin{array}{l}\sim 4.7 \\
(\sim 57)\end{array}$ \\
\hline $\begin{array}{l}\text { Pachyteuthis } \\
\text { optima }\end{array}$ & VSGM BP-11445 & $\begin{array}{l}\text { Sokur; Saratov } \\
\text { region } \mathrm{J}_{2} \mathrm{bt}_{1}\end{array}$ & 59.4 & $\begin{array}{c}10.5 \\
(100)\end{array}$ & $\begin{array}{l}10.2 \\
(97)\end{array}$ & $\begin{array}{c}45.3 \\
(431)\end{array}$ & 25 & 29 & $\begin{array}{c}5.5 \\
(100)\end{array}$ & $\begin{array}{c}5.4 \\
(98)\end{array}$ & $\begin{array}{c}0.8 \\
(15)\end{array}$ & $\begin{array}{l}2.9 \\
(53)\end{array}$ \\
\hline $\begin{array}{l}\text { Pachyteuthis } \\
\text { optima }\end{array}$ & VSGM BP-11446 & $\begin{array}{l}\text { Sokur; Saratov } \\
\text { region } \mathrm{J}_{2} \mathrm{bt}_{1}\end{array}$ & 125.5 & $\begin{array}{c}23.9 \\
(100)\end{array}$ & $\begin{array}{l}23.5 \\
(98)\end{array}$ & $\begin{array}{l}85.0 \\
(356)\end{array}$ & 24 & 20 & $\begin{array}{c}14.0 \\
(100)\end{array}$ & $\begin{array}{l}12.5 \\
(89)\end{array}$ & $\begin{array}{c}1.5 \\
(11)\end{array}$ & $\begin{array}{l}\sim 8.0 \\
(\sim 64)\end{array}$ \\
\hline $\begin{array}{l}\text { Pachyteuthis } \\
\text { optima }\end{array}$ & VSGM BP-11447 & $\begin{array}{l}\text { Sokur; Saratov } \\
\text { region } \mathrm{J}_{2} \mathrm{bt}_{1}\end{array}$ & 88.7 & $\begin{array}{c}20.2 \\
(100)\end{array}$ & $\begin{array}{c}20.2 \\
(100)\end{array}$ & $\begin{array}{l}\sim 78.0 \\
(\sim 386)\end{array}$ & 21 & - & - & 5.4 & $\begin{array}{c}0.9 \\
(\leq 17)\end{array}$ & $\begin{array}{l}3.0 \\
(56)\end{array}$ \\
\hline $\begin{array}{l}\text { Pachyteuthis } \\
\text { optima }\end{array}$ & VSGM BP-11448 & $\begin{array}{l}\text { Sokur; Saratov } \\
\text { region } \mathrm{J}_{2} \mathrm{bt}_{1}\end{array}$ & 65.0 & $\begin{array}{l}11.6 \\
(100)\end{array}$ & $\begin{array}{l}11.7 \\
(101)\end{array}$ & $\begin{array}{l}49.0 \\
(422)\end{array}$ & 26 & 23 & $\begin{array}{c}8.2 \\
(100)\end{array}$ & $\begin{array}{l}7.5 \\
(91)\end{array}$ & $\begin{array}{c}1.1 \\
(13)\end{array}$ & $\begin{array}{l}4.6 \\
(61)\end{array}$ \\
\hline $\begin{array}{l}\text { Pachyteuthis sp. } \\
\text { juv. }\end{array}$ & VSGM BP-11449 & $\begin{array}{l}\text { Sokur; Saratov } \\
\text { region } \mathrm{J}_{2} \mathrm{bt}_{1}\end{array}$ & 56.6 & $\begin{array}{c}9.55 \\
(100)\end{array}$ & $\begin{array}{c}9.55 \\
(100)\end{array}$ & $\begin{array}{l}42.7 \\
(447)\end{array}$ & 26 & 27 & $\begin{array}{c}5.3 \\
(100)\end{array}$ & $\begin{array}{l}4.8 \\
(91)\end{array}$ & $\begin{array}{c}0.7 \\
(13)\end{array}$ & $\begin{array}{l}\sim 2.6 \\
(\sim 54)\end{array}$ \\
\hline $\begin{array}{l}\text { Pachyteuthis sp. } \\
\text { juv. }\end{array}$ & VSGM BP-11450 & $\begin{array}{l}\text { Sokur; Saratov } \\
\text { region } \mathrm{J}_{2} \mathrm{bt}_{1}\end{array}$ & 56.7 & $\begin{array}{c}7.94 \\
(100)\end{array}$ & $\begin{array}{l}7.45 \\
(94)\end{array}$ & $\begin{array}{l}45.1 \\
(568)\end{array}$ & 28 & 24 & $\begin{array}{c}5.4 \\
(100)\end{array}$ & $\begin{array}{c}5.2 \\
(96)\end{array}$ & $\begin{array}{l}0.75 \\
(14)\end{array}$ & $\begin{array}{l}3.2 \\
(62)\end{array}$ \\
\hline $\begin{array}{l}\text { Holcobeloides } \\
\text { beaumontianus } \\
\text { (phragmocone) }\end{array}$ & VSGM BP-11451 & $\begin{array}{l}\text { Dubki; Saratov } \\
\text { region } \mathrm{J}_{2} \mathrm{k}_{3}-\mathrm{Ox}_{1}\end{array}$ & 15.4 & - & - & - & 17 & 17 & $\begin{array}{c}7.0 \\
(100)\end{array}$ & $\begin{array}{c}7.5 \\
(107)\end{array}$ & $\begin{array}{l}1.1 \\
(15)\end{array}$ & $\begin{array}{l}5.0 \\
(67)\end{array}$ \\
\hline $\begin{array}{l}\text { Holcobeloides } \\
\text { beaumontianus }\end{array}$ & VSGM BP-11452 & $\begin{array}{l}\text { Dubki; Saratov } \\
\text { region } \mathrm{J}_{2} \mathrm{k}_{3}-\mathrm{Ox}_{1}\end{array}$ & 110.9 & $\begin{array}{c}15.9 \\
(100)\end{array}$ & $\begin{array}{c}18.1 \\
(114)\end{array}$ & $\begin{array}{c}71.9 \\
(452)\end{array}$ & 20 & 19 & $\begin{array}{c}10.2 \\
(100)\end{array}$ & $\begin{array}{c}10.5 \\
(103)\end{array}$ & - & $\begin{array}{l}6.4 \\
(62)\end{array}$ \\
\hline $\begin{array}{l}\text { Cylindroteuthis } \\
\text { puzosiana }\end{array}$ & VSGM BP-11453 & $\begin{array}{l}\text { Dubki; Saratov } \\
\text { region } \mathrm{J}_{2} \mathrm{k}_{3}-\mathrm{ox}_{1}\end{array}$ & $>181$ & $\begin{array}{l}20.5 \\
(100)\end{array}$ & $\begin{array}{l}20.0 \\
(98)\end{array}$ & $\begin{array}{c}125 \\
(610)\end{array}$ & 17 & 17 & $\begin{array}{c}13.0 \\
(100)\end{array}$ & $\begin{array}{c}13.0 \\
(100)\end{array}$ & - & $\begin{array}{c}7.7 \\
(59)\end{array}$ \\
\hline $\begin{array}{l}\text { Cylindroteuthis } \\
\text { puzosiana }\end{array}$ & VSGM BP-11454 & $\begin{array}{l}\text { Dubki; Saratov } \\
\text { region } \mathrm{J}_{2} \mathrm{k}_{3}-\mathrm{Ox}_{1}\end{array}$ & 185 & $\begin{array}{c}24.0 \\
(100)\end{array}$ & $\begin{array}{l}23.9 \\
(100)\end{array}$ & $\begin{array}{c}134 \\
(558) \\
\end{array}$ & 19 & 21.5 & $\begin{array}{c}15.1 \\
(100) \\
\end{array}$ & $\begin{array}{l}14.3 \\
(95) \\
\end{array}$ & $(\overline{14})$ & $\begin{array}{c}8.8 \\
(60) \\
\end{array}$ \\
\hline \multicolumn{13}{|c|}{ Megateuthidid belemnites } \\
\hline $\begin{array}{l}\text { Paramegateuthis } \\
\text { timanensis }\end{array}$ & $\begin{array}{l}\text { VSGM VI-126/256 } \\
\text { (holotype, figured in } \\
\text { Gustomesov 1960a) }\end{array}$ & $\begin{array}{l}\text { Razlivnoi; Komi } \\
\mathrm{J}_{2} \mathrm{bt}_{1-? 2}\end{array}$ & 52.9 & $\begin{array}{c}13.4 \\
(100)\end{array}$ & $\begin{array}{l}11.9 \\
(89)\end{array}$ & $\begin{array}{l}33.0 \\
(246)\end{array}$ & 26 & 24 & $\begin{array}{c}8.47 \\
(100)\end{array}$ & $\begin{array}{l}7.95 \\
(94)\end{array}$ & - & - \\
\hline $\begin{array}{l}\text { Paramegateuthis } \\
\text { ishmensis }\end{array}$ & $\begin{array}{l}\text { VSGM VI-126/254 } \\
\text { (holotype, figured in } \\
\text { Gustomesov 1960a) }\end{array}$ & $\begin{array}{l}\text { Razlivnoi; Komi } \\
\mathrm{J}_{2} \mathrm{bt}_{1-? 2}\end{array}$ & 65.9 & $\begin{array}{c}12.5 \\
(100)\end{array}$ & $\begin{array}{l}11.0 \\
(88)\end{array}$ & $\begin{array}{l}43.0 \\
(345)\end{array}$ & 23.5 & 24 & $\begin{array}{c}9.39 \\
(100)\end{array}$ & $\begin{array}{l}8.55 \\
(91)\end{array}$ & - & - \\
\hline $\begin{array}{l}\text { "Nannobelus } \\
\text { bellus" }\end{array}$ & $\begin{array}{l}\text { VGSM BP-09666 } \\
\text { (CR 2790; holotype) }\end{array}$ & $\begin{array}{l}\text { Sokur; Saratov } \\
\text { region } \mathrm{J}_{2} \mathrm{bt}_{1}\end{array}$ & 65.5 & $\begin{array}{c}17.3 \\
(100)\end{array}$ & $\begin{array}{l}15.8 \\
(91)\end{array}$ & $\begin{array}{l}40.3 \\
(233)\end{array}$ & 32 & 31 & $\begin{array}{c}11.1 \\
(100)\end{array}$ & $\begin{array}{l}10.3 \\
(94)\end{array}$ & $\begin{array}{c}1.1 \\
(10)\end{array}$ & - \\
\hline $\begin{array}{l}\text { "Nannobelus } \\
\text { bellus" }\end{array}$ & $\begin{array}{l}\text { VGSM BP-09668 } \\
\text { (CR 2793; paratype) }\end{array}$ & $\begin{array}{l}\text { Sokur; Saratov } \\
\text { region } \mathrm{J}_{2} \mathrm{bt}_{1}\end{array}$ & 59.1 & $\begin{array}{c}15.2 \\
(100)\end{array}$ & $\begin{array}{l}14.1 \\
(93)\end{array}$ & $\begin{array}{l}30.3 \\
(199)\end{array}$ & 32 & 26 & $\begin{array}{c}8.2 \\
(100)\end{array}$ & $\begin{array}{l}7.35 \\
(90)\end{array}$ & $\begin{array}{c}1.0 \\
(13)\end{array}$ & $\begin{array}{l}3.3 \\
(44)\end{array}$ \\
\hline $\begin{array}{l}\text { "Nannobelus } \\
\text { bellus" }\end{array}$ & $\begin{array}{l}\text { VGSM BP-09667 } \\
\text { (CR 2792; paratype) }\end{array}$ & $\begin{array}{l}\text { Sokur; Saratov } \\
\text { region } \mathrm{J}_{2} \mathrm{bt}_{1}\end{array}$ & 45.6 & $\begin{array}{c}14.5 \\
(100)\end{array}$ & $\begin{array}{l}12.7 \\
(88)\end{array}$ & $\begin{array}{c}26.6 \\
(183)\end{array}$ & 28 & 29 & $\begin{array}{c}8.5 \\
(100)\end{array}$ & $\begin{array}{l}8.2 \\
(96)\end{array}$ & $\begin{array}{c}1.1 \\
(13)\end{array}$ & $\begin{array}{l}? 3.8 \\
(? 46)\end{array}$ \\
\hline $\begin{array}{l}\text { "Nannobelus } \\
\text { parabellus" }\end{array}$ & $\begin{array}{l}\text { VGSM BP-09670 } \\
\text { (CR 2791; holotype) }\end{array}$ & $\begin{array}{l}\text { Sokur; Saratov } \\
\text { region } \mathrm{J}_{2} \mathrm{bt}_{1}\end{array}$ & 55.4 & $\begin{array}{l}\sim 12.3 \\
(100)\end{array}$ & $\begin{array}{l}\sim 12.4 \\
(\sim 101)\end{array}$ & $\begin{array}{l}\sim 28.4 \\
(\sim 231)\end{array}$ & 26.5 & 28 & $\begin{array}{c}10.0 \\
(100)\end{array}$ & $\begin{array}{l}9.5 \\
(95)\end{array}$ & $\begin{array}{c}1.5 \\
(15)\end{array}$ & $\begin{array}{l}4.6 \\
(48)\end{array}$ \\
\hline $\begin{array}{l}\text { "Nannobelus } \\
\text { parabellus" }\end{array}$ & $\begin{array}{l}\text { VGSM BP-09672 } \\
\text { (CR 2795; paratype) }\end{array}$ & $\begin{array}{l}\text { Sokur; Saratov } \\
\text { region } \mathrm{J}_{2} \mathrm{bt}_{1}\end{array}$ & 38.2 & $\begin{array}{c}9.5 \\
(100)\end{array}$ & $\begin{array}{l}8.8 \\
(93)\end{array}$ & $\begin{array}{c}20.8 \\
(219)\end{array}$ & 34 & 34 & $\begin{array}{c}6.8 \\
(100)\end{array}$ & $\begin{array}{c}5.9 \\
(87)\end{array}$ & $\begin{array}{c}0.7 \\
(11)\end{array}$ & $\begin{array}{l}? 2.5 \\
(? 42)\end{array}$ \\
\hline $\begin{array}{l}\text { Megateuthididae } \\
\text { gen. et sp. nov. }\end{array}$ & VSGM BP-11458 & $\begin{array}{l}\text { India, Kachchh, } \\
\mathrm{J}_{2} \mathrm{bt}_{2}\end{array}$ & 17.1 & $\begin{array}{l}6.0^{* * *} \\
(100)\end{array}$ & $\begin{array}{l}5.7 * * \\
(94)\end{array}$ & - & 19 & 20 & - & - & - & - \\
\hline $\begin{array}{l}\text { Megateuthididae } \\
\text { gen. et sp. nov. }\end{array}$ & VSGM BP-11459 & $\begin{array}{l}\text { India, Kachchh, } \\
\mathrm{J}_{2} \mathrm{bt}_{2}\end{array}$ & 10.2 & $\begin{array}{c}6.0 \\
(100)\end{array}$ & $\begin{array}{c}5.3 \\
(88) \\
\end{array}$ & $\begin{array}{l}\sim 7.8 \\
(130)\end{array}$ & - & - & - & - & - & - \\
\hline
\end{tabular}


Table 1. continue

\begin{tabular}{|c|c|c|c|c|c|c|c|c|c|c|c|c|}
\hline \multirow[t]{2}{*}{ Taxon name } & \multirow{2}{*}{$\begin{array}{l}\text { Specimen No./ } \\
\text { reference to figured } \\
\text { material }\end{array}$} & \multirow{2}{*}{$\begin{array}{l}\text { Locality, region, } \\
\text { age }\end{array}$} & \multicolumn{4}{|c|}{ Rostrum } & \multicolumn{5}{|c|}{ Phragmocone } & \multirow{2}{*}{\begin{tabular}{|c} 
Proostracum \\
$\mathrm{w}, \mathrm{mm}$ \\
$(\%)$ from $1 \mathrm{l}(\mathrm{p})$
\end{tabular}} \\
\hline & & & $\mathrm{R}, \mathrm{mm}$ & $\begin{array}{c}\mathrm{DV}, \\
\mathrm{mm}(\%)\end{array}$ & $\begin{array}{c}\mathrm{LL}, \\
\mathrm{mm}(\%)\end{array}$ & $\begin{array}{c}\mathrm{PA} \\
\mathrm{mm}(\%)\end{array}$ & $\beta(\mathrm{dv})^{\circ}$ & $\beta(11)^{\circ}$ & $\begin{array}{c}\mathrm{dv}(\mathrm{p}) \\
(\%)\end{array}$ & $\begin{array}{l}11(\mathrm{p}) \\
(\%)\end{array}$ & $\begin{array}{c}\mathrm{cl}, \mathrm{mm} \\
(\%)\end{array}$ & \\
\hline \multicolumn{13}{|c|}{ Belemnotheutididae } \\
\hline $\begin{array}{l}\text { Acanthoteuthis } \\
\text { antiqua }\end{array}$ & VSGM BP-11455 & $\begin{array}{l}\text { Dubki; Saratov } \\
\text { region } \mathrm{J}_{2} \mathrm{k}_{3}-\mathrm{Ox}_{1}\end{array}$ & 37 & - & - & - & 20 & 21 & $10.2 *$ & $10.3^{*}$ & $\begin{array}{c}1.4 \\
(14)\end{array}$ & $\begin{array}{l}5.1 \\
(50)\end{array}$ \\
\hline $\begin{array}{l}\text { Acanthoteuhtis } \\
\text { antiqua }\end{array}$ & VSGM BP-11456 & $\begin{array}{l}\text { Dubki; Saratov } \\
\text { region } \mathrm{J}_{2} \mathrm{k}_{3}-\mathrm{ox}_{1}\end{array}$ & 30.6 & - & - & - & 19 & 20 & $7.3^{*}$ & $7.3^{*}$ & $\begin{array}{c}1.2 \\
(16)\end{array}$ & - \\
\hline $\begin{array}{l}\text { Acanthoteuthis } \\
\text { antiqua }\end{array}$ & VSGM BP-11457 & $\begin{array}{l}\text { Dubki; Saratov } \\
\text { region } \mathrm{J}_{2} \mathrm{k}_{3}-\mathrm{ox}_{1}\end{array}$ & 27.4 & - & - & - & 17 & 17 & - & $6.5^{*}$ & $\begin{array}{c}1.4 \\
(16) \\
\end{array}$ & $\begin{array}{l}3.3 \\
(51) \\
\end{array}$ \\
\hline \multicolumn{13}{|c|}{ Other morphologically comparable coleoids } \\
\hline $\begin{array}{l}\text { Diplobelus } \\
\text { belemnitoides }\end{array}$ & $\begin{array}{l}\text { (Jeletzky } 1981 \text {; } \\
\text { pl. } 25, \text { fig. } 1 b ; \\
\text { paratype) }\end{array}$ & $\begin{array}{l}\text { Stramberg; } \\
\text { Czech Rep. } \mathrm{J}_{3} \mathrm{t}_{3}\end{array}$ & 34.8 & $\begin{array}{c}6.5 \\
(100)\end{array}$ & $\begin{array}{c}5.8 \\
(89)\end{array}$ & $\begin{array}{l}3.0 \\
(46)\end{array}$ & 25 & - & $\begin{array}{c}5.2 \\
(100)\end{array}$ & - & $\begin{array}{c}1.0 \\
(19)\end{array}$ & - \\
\hline $\begin{array}{l}\text { Diplobelus } \\
\text { belemnitoides }\end{array}$ & (Naef 1922, fig. 65i) & $\begin{array}{l}\text { Stramberg; } \\
\text { Czech Rep. } \mathrm{J}_{3} \mathrm{t}_{3}\end{array}$ & - & - & - & - & - & 23 & - & $(100)$ & - & $(29)$ \\
\hline $\begin{array}{l}\text { Pavloviteuthis } \\
\text { cantiana }\end{array}$ & $\begin{array}{l}\text { (Jeletzky 1981; pl. } \\
\text { 23; holotype) }\end{array}$ & $\begin{array}{l}\text { Folkestone, } \\
\text { England } \mathrm{K}_{1} \mathrm{a}_{1}\end{array}$ & 15.1 & - & - & - & - & 27 & - & $\begin{array}{c}5.8^{*} \\
(100)\end{array}$ & $\begin{array}{c}0.8 \\
(13)\end{array}$ & $\begin{array}{c}- \\
(29)\end{array}$ \\
\hline $\begin{array}{l}\text { Tauriconites } \\
\text { nikolai }\end{array}$ & $\begin{array}{l}\text { (Drushtchits et al. } \\
\text { 1984; pl. I, fig. 1a) }\end{array}$ & $\begin{array}{l}\text { Balki, Crimea } \\
\mathrm{K}_{1} \mathrm{~b}\end{array}$ & - & - & - & - & - & 21 & $\begin{array}{c}4.2 \\
(100)\end{array}$ & - & $\begin{array}{l}1.1 \\
(26)\end{array}$ & - \\
\hline $\begin{array}{l}\text { Tauriconites } \\
\text { nikolai }\end{array}$ & $\begin{array}{l}\text { (Drushtchits et al. } \\
\text { 1984; pl. I, fig. 2; } \\
\text { holotype) }\end{array}$ & $\begin{array}{l}\text { Letnee, Crimea } \\
\mathrm{K}_{1} \mathrm{~b}\end{array}$ & - & - & - & - & - & 17 & $\begin{array}{c}2.9 \\
(100)\end{array}$ & - & $\begin{array}{c}1.0 \\
(34)\end{array}$ & - \\
\hline
\end{tabular}

(in the paratype) margins. No apical part can be clearly separated. Apex is central.

Sculpture: Represented by a single dorsal ridge, running throughout the whole rostrum. This ridge is strongly expressed in the apical part of the rostrum, where its elevation attains totally $1 / 5$ of dorsoventral diameter in the same section, decreasing adorally to $1 /{ }_{12}-1 / 15$, with a tendency to disappear at the anterior end of the alveolar part. The configuration of growth lines fully supports this idea, showing no dorsal ridge in the cross-section at the anterior broken end of the rostrum. The width of ridge remains uniform throughout the whole length of the rostrum, and slightly narrows only near the apex. The cross-section of the dorsal ridge is depressed semi-circular throughout all its length.

Surface of the rostrum: Shows fine longitudinal striae running adapically. The striae are most pronounced to the sides of dorsal ridge. They are short and widely spaced on lateral sides and on the dorsal ridge, while to the sides of the dorsal ridge and on the ventral side of the rostrum they are densely spaced and are stretching through the whole postalveolar part. Microscopic examination shows that the striae can dichotomize.

Alveolus: Has circular cross-section and occupies more than half of the rostrum (no anterior part preserved in our material), the phragmocone angle is $16-18^{\circ}$. No original matter of conotheca or septa is preserved but the configura- tion of septal lines can be read by unclear color imprints on the alveolus walls. The camera length is about $0.12-0.14$ of the corresponding phragmocone diameter. The septal lines seem to be in general straight, with no dorsal saddle. On the lateral sides they slightly turn rearwards when approaching the ventral side. The alveolus is symmetrical in dorso-ventral section, with the tip located subcentrally. Configuration of growth lines can be easily traced on the alveolus wall, but additionally alveolus bears microsculpture looking as minute striation, best seen on the ventral (longitudinal) and ventro-lateral (oriented obliquely upwards-forward) sides.

Proostracum: Observed in the holotype as imprint on dorsal wall of the alveolus, wide ( 0.51 of the corresponding phragmocone lateral diameter), and having blunt spatulate-like anterior margin. Three thin longitudinal ribs are well seen - the central unpaired one, slightly transforming to a narrow flattened field adorally, and two uniform dorsolateral, limiting central field of the proostracum. Lateral fields are relatively narrow, their width attaining $1 / 5$ of central field each.

Measurements: See Table 1.

Remarks. - X-ray diffraction analysis, performed for the holotype rostrum fragment at anterior end, has shown it to be $100 \%$ calcitic. Considering the fact that ammonites in corresponding clayish Kimmeridgian layers usually 
demonstrate preserved nacre (= aragonitic matter), calcitic mineralogy of Chuvashiteuthis aenigmatica gen. et sp. nov. rostra is evidently a primary character.

Occurrence. - Known only from its type horizon in Chuvash Republic (Central Russia).

\section{Discussion}

\section{Systematic position}

Chuvashiteuthis aenigmatica gen. et sp. nov. is stratigraphically isolated from other Megateuthididae, which are considered to completely decline during the Bathonian (Dzyuba \& Weis 2015, Dzyuba et al. 2015, see below) probably as a result of evolutionary pressure of more progressive taxa (Cylindroteuthididae, Belemnopseidae; Ippolitov \& Desai unpublished data). Chuvashiteuthis gen. nov. demonstrates a combination of characters, which makes necessary to provide some justification for its placement inside the family Megateuthididae.

There is number of morphologically comparable taxa among fossil Mesozoic coleoids, some of which can be potentially found in the upper Kimmeridgian of the Russian Platform. Biometrical comparison of Chuvashiteuthis aenigmatica gen. et sp. nov. with the taxa discussed below can be obtained from Table 1 .

Diplobelids and other possibly related taxa. - Among Late Jurassic to Early Cretaceous members of the order Diplobelida, the only genus Diplobelus Naef, 1926 from the Tithonian is similar to Chuvashiteuthis gen nov. by the shape of its rostrum (also called "sheath", see Fuchs 2012) and by the presence of some kind of dorsal narrowing of the rostrum (von Zittel 1868, fig. 14i), while some other taxa [Albian "Pavloviteuthis" cantiana (Spath, 1939) see Jeletzky 1981; Berriasian-lower Valanginian Tauriconites nikolai Kabanov (in Drushtchits et al. 1984)] demonstrate similar shape only. The main apomorphies of diplobelids are narrowed proostracum and the presence of dorsal saddle on suture lines (Jeletzky 1981). Despite few is still clear about the nature of diplobelids and their precise position among Coleoidea (e.g. Ippolitov et al. 2010), as well as about real weight of diplobelid "apomorphies", the proostracum in Chuvashiteuthis aenigmatica gen. et sp. nov. seems to be wide and reliably "spatulate" (sensu Doyle \& Shakides 2004), (wp = 51\% in the holotype), and therefore differs much from comparable Diplobelidae ( $w p=29 \%$ in both Pavloviteuthis and Diplobelus), including the type genus of the family. From Tauriconites, which lacks data about proostracum width, $C h$. aenigmatica gen. et sp. nov. clearly differs by shorter camera $(\mathrm{cl}=14 \%$ vs $26-34 \%$ in Tauriconites nikolai). Ch. aenigmatica gen. et sp. nov. also does not demonstrate any traces of dorsal saddle on the septal line.

Belemnotheutids. - Genus Acanthoteuthis Wagner (in von Münster 1839), which is the most known and widely distributed member of the order Belemnotheutida Stolley, 1919 (or suborder - see Doyle \& Shakides 2004), has narrow dorsal ridge running adapically on the fragile sheathlike aragonitic rostrum (Bandel \& Kulicki 1988, Doyle \& Shakides 2004). Belemnotheutids, comprising the single family Belemnotheutididae von Zittel, 1884, occur in the Callovian and more rarely in the Late Jurassic of Central Russia (Rogov \& Bizikov 2008, fig. 3), but are still poorly studied. They look especially attractive as possible Chuvashiteuthis gen. nov. relatives not only because of similar rostrum shape and longitudinal rostral structure, well-comparable with that of Chuvashiteuthis gen. nov., but also by having narrow phragmocone angles (see Rogov \& Bizikov 2008, fig. 1), characteristic for Ch. aenigmatica gen. et sp. nov., too.

From the biometrical comparison, it can be seen that principal phragmocone characters of Chuvashiteuthis gen. nov. (proostracum width and latest camerae lengths) are well-comparable with those of Acanthoteuthis antiqua (Pearce, 1847). However Chuvashiteuthis aenigmatica gen. et sp. nov. has fully calcitic rostrum, which is more or less massive, while all belemnotheutids known to possess thin aragonitic rostrum. Additionally, in all known cases rostra of Acanthoteuthis demonstrate bifurcating keel, while in Chuvashiteuthis gen. nov. the keel is simple. Nevertheless, considering high similarity of phragmocones, affinity to Belemnotheutididae is the first feasible hypothesis to be discussed below.

Belemnitids or "true" belemnites. - Two belemnite families can be considered as potential candidates for testing the affinity of Chuvashiteuthis gen. nov. The first is Middle Jurassic to Early Cretaceous Cylindroteuthididae, which are the commonest and most diversified belemnites in the Boreal Realm and among all, have produced short subconical forms in their evolution. The second is Early-Middle Jurassic Megateuthididae, which are essentially similar by the general shape of the rostrum, but are considered to die out during the Bathonian (Dzyuba \& Weis 2015, Dzyuba et al. 2015).

Relative length of the camerae in Chuvashiteuthis aenigmatica gen. et sp. nov. $(\mathrm{cl}=14 \%)$ is well comparable both with megateuthidids $(\mathrm{cl}=10-15 \%)$ and cylindroteuthidids $(\mathrm{cl}=11-15 \%)$; while relative proostracum width ( $\mathrm{pw}=51 \%)$ is somewhat lying between measured Megateuthididae $(\mathrm{pw}=44-48 \%)$ and Cylindroteuthididae (pw $=53-66 \%$ ). Alveolar angle in Chuvashiteuthis aenigmatica gen. et sp. nov. (16-18 $)$ is comparable with certain members of both families. Similar state of character 


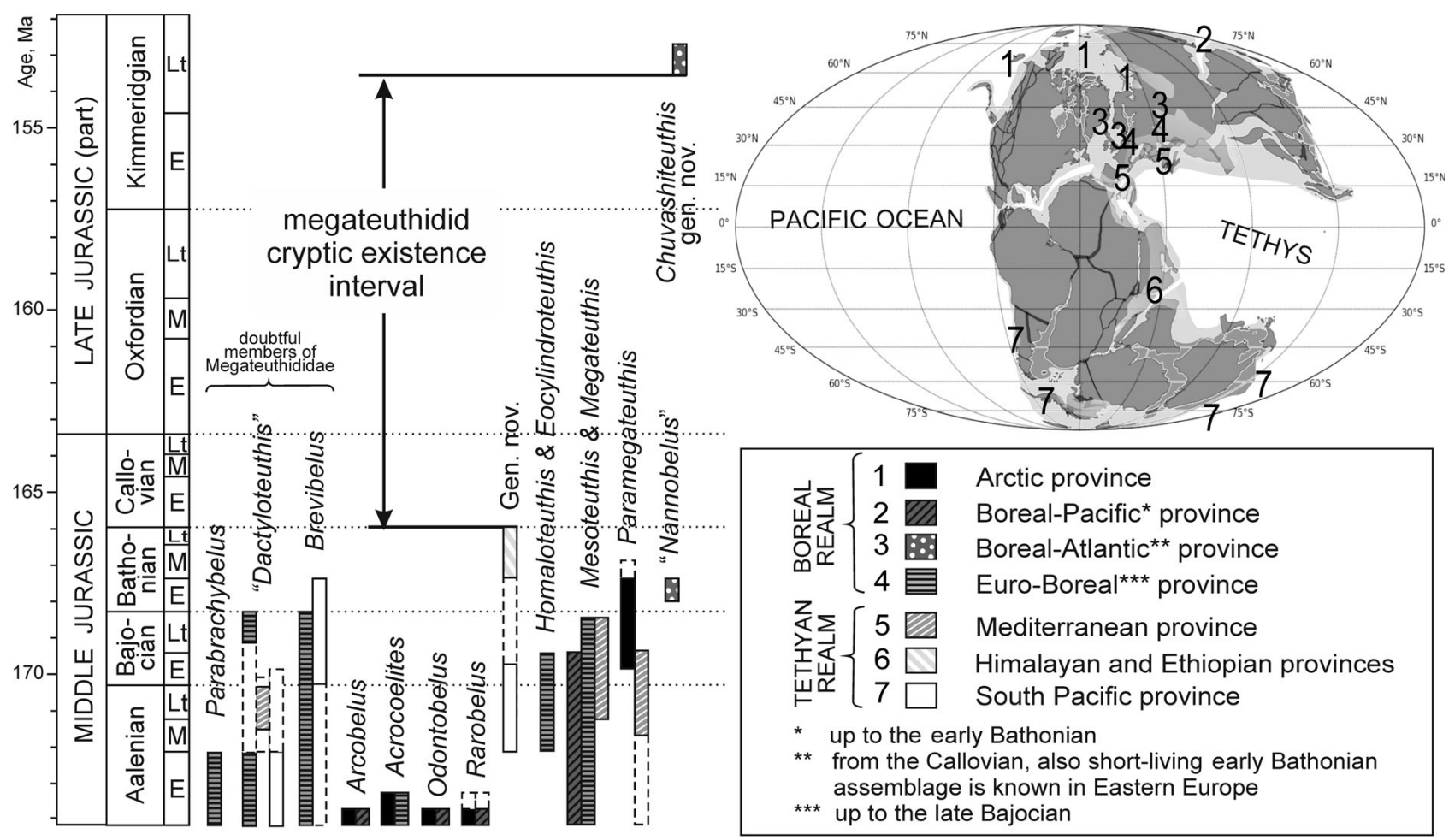

Figure 6. Spatial and temporal distribution of Middle-Late Jurassic megateuthidids illustrating the 12 Ma gap between Chuvashiteuthis gen. nov. and previous records. Geological time scale is based on Ogg et al. (2016). Palaeogeographical basis is by Palaeomap maker software at GPlates Portal (portal.gplates.org) for the age $167 \mathrm{Ma}$ (mid-Bathonian) with epicontinental seas drawn after Scotese (2016) data. Palaeobiogeographical subdivision is based on terminology and nomenclature employed by Challinor et al. (1992), with addition of "Euro-Boreal" province, first introduced by Dommergues (1987) for the European Lower Jurassic ammonite assemblages and widely employed in Recent belemnite papers (Dera et al. 2016, Weis et al. 2017) as a synonym of Boreal-Atlantic belemnite province of Challinor et al. (1992). Despite both biochoremas occupy similar territories, it looks reasonable to split them chronologically, restricting the Boreal-Atlantic province only to Callovian and above, and Euro-Boreal province - to Lower and early Middle Jurassic. The crucial difference between these biochoremas is that their belemnite biota is not successive: from Callovian onwards the assemblage demonstrate clear Arctic affinities at generic level, while all the major taxa characterizing the Aalenian-Bajocian assemblages (Brevibelus Doyle, 1992; Homaloteuthis Stolley, 1919; Eocylindroteuthis Riegraf, 1980) often associate with Tethyan elements in ecotone areas, but are absent in Arctic basins, indicating strong isolation from high latitudes and autonomous development of belemnite biota along the northern Tethyan margin. Vertical distribution of genera is according to Dzyuba \& Weis (2015) and Weis et al. (2017) with additions from Doyle et al. (1996), Challinor (2000), Challinor \& Hikuroa (2007) and A.P.I. unpublished data.

is shared by Cylindroteuthididae with elongated rostrum [17-21.5 ${ }^{\circ}$ were obtained for Holcobeloides beaumontianus (d'Orbigny, 1842) and Cylindroteuthis puzosiana (d'Orbigny, 1842)]. Pachyteuthis spp. with less elongated rostra were found to have larger alveolar angle (19-29 $)$. For megateuthidids, we recorded $20-34^{\circ}$ and this is still larger, than in Chuvashiteuthis gen. nov. Also worth mentioning, that some of described Siberian species of Paramegateuthis [P. nescia Nalnjaeva in Sachs \& Nalnjaeva (1975) and P. manifesta Nalnjaeva in Sachs \& Nalnjaeva (1975)] demonstrate very closely spaced dorso-lateral furrows, so that its dorsal area is restricted by these furrows (Sachs \& Nalnjaeva 1975, pl.9, fig. 3.5; de Lagausie \& Dzuyba 2017, pl. 1, figs 22-24) and resembling the "keel" of Chuvashiteuthis gen. nov. Undescribed Indian megateuthidids from the middle-late Bathonian (Ippolitov \& Desai unpublished data) are essentially similar to Paramegateuthis by their alveolar angle (19-20 ${ }^{\circ}$ and general shape, but have angular cross-section and wider spaced furrows, than in Chuvashiteuthis gen. nov.

Proposed affinity. - Detailed phragmocone study allows to override the similarity with Diplobelidae, but does not provide any reliable key for further solving the affinity of Chuvashiteuthis gen. nov. Three different taxa (Cylindroteuthididae, Megateuthididae, Belemnotheutididae) demonstrate similar characters of phragmocones, that in turn may reflect close phylogenetic relationship between these taxa. Consequently, only rostral characters can be used for discussing the affinity. The reasons, validating our placement of Chuvashiteuthis aenigmatica gen. et sp. nov. into the family Megateuthididae, are as following: (1) conical rostrum shape with lateral compression, typical for many later (Bajocian-Bathonian) megateuthidids, including the type genus of the family; (2) dorsal keel, which is restricted by 
dorsolateral furrows and can be considered as probably homologous to dorsal area of megateuthidids; (3) rostrum made of calcite, like in normal belemnites (Megateuthididae and Cylindroteuthididae), while in Belemnotheutididae the rostrum is aragonitic.

Being included into Megateuthididae, Chuvashiteuthis aenigmatica gen. et sp. nov. becomes the youngest record of the family, and widens known stratigraphical distribution of megateuthidid belemnites to the upper Kimmeridgian, extending the total range of the group by $\sim 12$ million years.

\section{Relation to other megateuthidids}

Spatial and temporal distribution of late megateuthidids is illustrated in Fig. 6. Stratigraphically the youngest megateuthidid genus hitherto known in Northern Hemisphere is Paramegateuthis Gustomesov, 1960a, which starts in the Tethyan Aalenian (Stoyanova-Vergilova 1983) and is widely distributed in the Late Bajocian-Bathonian (de Lagausie \& Dzuyba 2017), being restricted that time to high latitudes of the Northern Hemisphere.

The latest records of Paramegateuthis are from northern Siberia and Pechora Basin (northern part of European Russia and northern Siberia). Despite the fact that these finds were commonly referred as early Callovian (e.g. Gustomesov 1960a, Sachs \& Nalnjaeva 1975), newest detailed biostratigraphic re-investigation has re-dated their age as probably the early-?middle Bathonian (Mitta et al. 2015, de Lagausie \& Dzyuba 2017). These forms, named P. ishmensis and P. timanensis (Fig. 5E, F), differ from Chuvashiteuthis gen. nov. by weakly incised dorso-lateral grooves and well-defined apical part, which is indistinct in Chuvashiteuthis gen. nov.

From the lower Bathonian of the same territory as Chuvashiteuthis gen. nov. finds, there are also records of some peculiar forms originally described as Nannobelus (Barskov in Mitta et al. 2004), but later reconsidered as Paramegateuthis (Dzyuba in Mitta et al. 2014). Besides absence of dorsolateral grooves, these forms demonstrate extremely wide phragmocone angles (Tab. 1), not comparable with those in Chuvashiteuthis gen. nov.

Besides Paramegateuthis and "Nannobelus", which were restricted to Northern Hemisphere during the Bathonian, there are poorly known megateuthidids recorded from the middle-late Bathonian of India (Spath 1927, Ippolitov \& Desai unpublished data), which indicate the simultaneous presence of isochronous megateuthidids of the Bathonian age in the Tethyan Realm, at the northern margin of Gondwana. These megateuthidids (Fig. 5C, D) resemble juvenile Megateuthis and are characterized by tiny size and deeply incised dorsolateral grooves, running over the most part of the rostrum, but again diverging and not forming the narrow keel like in Chuvashiteuthis gen. nov. Their alveolar angles range mainly between 19 and $23^{\circ}$ and therefore are comparable with Chuvashiteuthis gen. nov.

From the first look Chuvashiteuthis gen. nov. demonstrates similarity with Tethyan forms from India mentioned above by its more prominent apical furrows, than in Paramegateuthis. But this character is often unstable within genera (Sachs \& Nalnjaeva 1975), and the cross-section of the newly described taxon is rounded with concave lateral sides showing no lateral lines or flattenings. Such one is characteristic for Paramegateuthis, while Indian rostra tend to have angular cross-section at least in adults. To conclude, the affinity with Paramegateuthis looks evident, but there is no certain species of Paramegateuthis which could be linked directly with Chuvashiteuthis aenigmatica gen. et sp. nov.

\section{Biogeographical interpretation}

In most cases sudden appearance of any faunal elements at narrow intervals can be interpreted as short-time invasions from neighbouring biogeographic province. The significant question about Chuvashiteuthis gen. nov. is where exactly was the refugium of megateuthidids located during the Callovian-Kimmeridgian time interval.

Belemnites, co-occurring in the upper Kimmeridgian with Chuvashiteuthis aenigmatica gen. et sp. nov. belong to the Boreal family Cylindroteuthididae, however, the complex is extremely poor and comprises the single species Boreioteuthis troslayana, mostly represented by tiny-sized rostra. These small-sized rostra were first recognized by Gustomesov (1960b, 1964), who assigned them to a new species, Pachyteuthis (Microbelus) gorodischensis Gustomesov, 1960b. In the most recent revision (Dzyuba 2004), this species was synonymised with Belemnites troslayanus d'Orbigny, 1850, widely distributed over the Boreal Realm, and the latter was assigned (Dzyuba 2004) to Boreioteuthis Sachs et Nalnyaeva, 1966 [subgenus of Pachyteuthis Bayle, 1878 after Dzyuba (2004), and a separate genus after Dzyuba (2011)]. Leaving behind the scope of the present paper the validity of this synonymization, it should be noted that Siberian representatives of the latter species are characterized by medium-sized rostra, exceeding $10 \mathrm{~mm}$ in diameter, and therefore specimens from the Russian Platform, which are usually about 5-6 mm only, can be interpreted as juvenile or ecologically depressed.

The total appearance of late Kimmeridgian complex accompanying Chuvashiteuthis gen. nov. finds also differs much from that of Northern Siberia and northern part of European Russia, where cylindroteuthidid belemnites are much more diverse and larger in size (e.g. see Dzyuba 2004). The peculiarities of belemnite complex, discussed 
above, may indicate some warming episode during the late Kimmeridgian in Central Russia, which oppressed the development of diversified cold-preferent cylindroteuthidid fauna.

Ammonite assemblages of the upper Kimmeridgian in Chuvashia as well as in other Middle Volga sections include Subboreal, Boreal and Tethyan faunal elements, and their relative abundance can also provide some key about climatic fluctuations. It should be noted that in all well-studied sections on the Russian Platform Tethyan ammonites, especially aspidoceratids, are more numerous comparing with Boreal taxa within the suspected interval with Chuvashiteuthis gen. nov. (Fig. 3). Such predominance of Tethyan ammonites is well-corresponding with scarce stable isotope data derived from belemnite rostra (Price \& Rogov 2009, Wierzbowski et al. 2013), showing the warming event during the mid-late Kimmeridgian at the studied area. This episode was also recognized in other Boreal regions, i.e. Subpolar Urals (Zakharov et al. 2005) and Northern Siberia (Zakharov et al. 2014). The hypothesis of strong Tethyan influence on Russian platform during the Kimmeridgian is supported by the similar drastic fall of belemnite diversity recorded in Europe and known as "Kimmeridgian crisis" and also correlated with warming event (Dera et al. 2016).

Considering the above, invasion from the north can be reasonably excluded for Chuvashiteuthis aenigmatica gen. et sp. nov. - as species-rich and highly diversified northern belemnite associations of this age do not penetrate to Central Russia.

Therefore, either Megateuthididae had a refugium since the Bathonian somewhere in the ecotone zone between Boreal and Tethyan realms, being superseded from their preferable habitat area at the north by quickly diversifying cylindroteuthidids, or had been existing in Middle Russian sea during the Callovian to late Kimmeridgian as a rare and still cryptic element of the biota. However, the latter assumption looks unlikely, as belemnite assemblages of the Callovian-Oxfordian of the Russian Platform, despite relatively well-studied (e.g. Gustomesov 1964), do not contain any megateuthidid records.

\section{Conclusion}

Chuvashiteuthis aenigmatica gen. et sp. nov., described herein from the upper Kimmeridgian of Central Russia, is a relict representative of the belemnite family Megateuthididae, which was thought to decline during the Bathonian. Its sudden appearance in the upper Kimmeridgian of Chuvashia is probably due to some warming, stressing the development of normal cylindroteuthidid faunas in Middle Russian sea. The precise location of megateuthidid refugium during the Callovian-early Kimmeridgian remains uncertain, but most likely it was located somewhere in the Boreal-Tethyan ecotone or even at the northern Tethyan margin, while the appearance in Central Russia is possibly a short-time invasion event.

\section{Acknowledgements}

The authors are grateful to V.L. Kosorukov (Moscow State University, Moscow) for his help with X-ray diffraction analysis of the samples. We also thank our reviewers O.S. Dzyuba (Trofimuk Institute of Petroleum Geology and Geophysics, Novosibirsk) for her very careful review and numerous remarks which helped to greately improve the manuscript, and G. Dera (Université Paul Sabatie, Toulouse) for his suggestions improving the general structure of the paper. The investigation was performed accordingly to the FANO investigation program no. 0135-2014-0064 of the Geological institute of RAS and supported by RFBR grants No. 15-05-06183, 15-05-03149, 15-55-45095 and DST project No. INT/RUS/RFBR/P-206.

\section{References}

BANDEL, K. \& KULICKI, C. 1988. Belemnoteuthis polonica: a belemnite with an aragonitic rostrum, 303-316. In WiedMann, J. \& Kullmann, J. (eds) Cephalopods - Present and Past. Schweitzerbart, Stuttgart.

BATHER, F.A. 1888. Shell-growth in cephalopods (Siphonopoda). Annales and Magazine of natural History (series 6) 1, 298-310.

BAyle, E. 1878. Fossiles principaux des terrains. In BAYLE, E. \& ZeILler, R. (eds) Explication de la carte géologique de France, T. 4, Atlas, Pt. 1. Imprimérie Nationale, Paris.

Challinor, A.B. 2000. Belemnoids, 323-326. In Grant-Mackie, J.A., Aita, Y., Balme, B.E., Challinor, A.B., MacFarlan, D.A.B., Molnar, R.E., Stevens, G.R. \& Thulborn, R.A. (eds) Jurassic palaeobiogeography of Australasia. Memoires of the Association of Australasian Palaeontologists 23, 311-354.

Challinor, A.B. \& Hikuroa, D.C.H. 2007. New Middle and Upper Jurassic Belemnite Assemblages from West Antarctica (Latady Group, Ellsworth Land): Taxonomy and Paleobiogeography. Palaeontologia Electronica 10(1), 6A: 29 p.

Challinor, A.B., Doyle, P., Howlett, P.J. \& Nal'nyaeva, T.I. 1992. Belemnites of the circum-Pacific region, 334-341, 636-645. In Westermann, G.E.G. (ed.) The Jurassic of the Circum-Pacific. Cambridge University Press, Cambridge.

Dera, G., Toumoulin, A., De Baets, K. 2016. Diversity and morphological evolution of Jurassic belemnites from South Germany. Palaeogeography, Palaeoclimatology, Palaeoecology 457, 80-97. DOI 10.1016/j.palaeo.2016.05.029

Dommergues, J.L. 1987. L'évolution chez les Ammonitina du Lias Moyen (Carixian, Domérien basal) en Europe 
occidentale. Documents des Laboratoires de Géologie Lyon 98, 1-297.

Doyle, P. 1992. The British Toarcian (Lower Jurassic) Belemnites. Part 2. Monograph of the Palaeontographical Society, London 587, 50-79, pls 18-28.

Doyle, P. \& Shakides, E.V. 2004. The Jurassic Belemnite Suborder Belemnotheutina. Palaeontology 47, 983-998. DOI 10.1111/j.0031-0239.2004.00395.x

Doyle, P., Kelly, S.R.A., Pirrie, D., Riccardi, A.C. \& Oliveiro, E. 1996. Belemnite biostratigraphy of the Southern Hemisphere: a comparative study of Antarctica and Argentina. Revista de la Asociación Geológica Argentina 51(4), 331-338.

Drushtchits, V.V., Kabanov, G.K. \& Nerodenko, V.M. 1984. Structure of the phragmocone and the rostrum in Tauriconites gen. nov. (Coleoidea, Diplobelida). Paleontologicheskij Zhurnal 1984(1), 12-18. [in Russian]

Dzyuba, O.S. 2004. Belemnites (Cylindroteuthidae) and biostratigraphy of the Middle and Upper Jurassic of Siberia. 203 pp. Publishing House of SB RAS, Department "GEO”, Novosibirsk. [in Russian with English summary]

Dzyuba, O.S. 2011. Subfamily classification within the family Cyindroteuthididae (Belemnitida). News of Paleontology and Stratigraphy 16-17, 103-108. (supplement to journal "Geologiya i Geofizika") [in Russian with English summary]

DzyuBa, O.S. 2012. Belemnites and Biostratigraphy of the Jurassic-Cretaceous Boundary Deposits of Northern East Siberia: New Data on the Nordvik Peninsula. Stratigraphy and Geological Correlation 20(1), 53-72.

DOI 10.1134/S0869593811060037

Dzyuba, O.S. \& WeIs, R. 2015. Megateuthididae (Belemnitina) from the Lower-Middle Jurassic of Northern and Eastern Russia: taxonomy and nomenclature, 62-64. In LeONOva, T.B., Barskov, I.S. \& MitTA, V.V. (eds) Contributions to current cephalopod research: Morphology, Systematics, Evolution, Ecology and Biostratigraphy. Proceeding of conference (Moscow, 2-4 April, 2012). Russian Academy of Sciences, Borissiak Paleontological Institute, Moscow. [in Russian with English abstract]

Dzyuba, O.S., Weis, R., Nalnjaeva, T.I. \& Riegraf, W. 2015. Rarobelus nom. nov. from the Boreal Toarcian-Aalenian and its systematic position (Belemnitida, Belemnitina, Megateuthididae). Neues Jahrbuch für Geologie und Paläontologie, Abhandlungen 275(3), 305-315. DOI 10.1127/njgpa/2015/0465

FuCHs, D. 2012. The "rostrum"-problem in coleoid terminology an attempt to clarify inconsistencies. Geobios 45, 29-39. DOI 10.1016/j.geobios.2011.11.014

GŁowniak, E., Kiselev, D.N., Rogov, M., Wierzbowski, A. \& Wright, J. 2010. The Middle Oxfordian to lowermost Kimmeridgian ammonite succession at Mikhalenino (Kostroma District) of Russian Platform, and its stratigraphical and palaeogeographical importance. Volumina Jurassica VIII, 8-45.

Gustomesov, V.A. 1960a. New Callovian belemnites from the Timan, 190-192. In MARKovsky, B.P. (ed.) New species of fossil plants and invertebrates of the USSR. Part II. Gosgeoltechizdat, Moscow. [in Russian]

Gustomesov, V.A. 1960b. Upper Jurassic and Valanginian belemnites of the European part of the USSR and Northern Trans-Urals, 195-210. In Markovsky, B.P. (ed.) New species of fossil plants and invertebrates of the USSR. Part II. Gosgeoltechizdat, Moscow. [in Russian]

Gustomesov, V.A. 1961. To the ecology of Late Jurassic belemnites (climatic zonality in distribution, lifemode, mass intravital injuries. Trudy Moskovskogo Geologorazvedochnogo instituta imeni S. Ordzhonikidze 37, 190-204. [in Russian]

Gustomesov, V.A. 1964. Boreal Late Jurassic belemnites (Cylindroteuthinae) of the Russian platform, 89-216. In Mikhailov, N.P. \& Gustomesov, V.A. (eds) Boreal Late Jurassic Cephalopods. Academy of Sciences of the USSR, Geological Institute, Transactions 107. [in Russian]

Ippolitov, A.P., Bizikov, V.A. \& Rogov, M.A. 2010. Chalalabelus finds from the Middle Volgian of Moscow city and the nature of enigmatic Diplobelida, 53. $8^{\text {th }}$ International Symposium, Cephalopods - Present and Past. August 30 September 3, 2010. Abstracts Volume. University of Burgundy \& CNRS, Dijon.

JELETZKY, J.A. 1966. Comparative morphology, phylogeny and classification of fossil Coleoidea. Paleontological Contributions, University of Kansas, Mollusca 7, 1-166.

JELETZKY, J.A. 1981. Lower Cretaceous diplobelinid belemnites from the Anglo-Paris Basin. Palaeontology 24(1), 115-145.

Kutek, J. \& Zeiss, A. 1997. The highest Kimmeridgian and Lower Volgian in Central Poland; their ammonites and biostratigraphy. Acta Geologica Polonica 47(3-4), 107-198.

Lagausie, B. de \& Dzyuba, O.S. 2017. Biostratigraphy of the Bajocian-Bathonian boudary interval in northern Siberia: new data on belemnites from the Yuryung-Tumus peninsula. Bulletin de la Société géologique de France 188 (1-2), 1-9. DOI 10.1051/bsgf/2017004

Lissajous, M. 1915. Quelques remarques sur les bélemnites jurassiques. Bulletin de la Société d'histoire naturelle de Mâcon 6, 1-32.

Mitta, V.V., Barskov, I.S., Gründel, J., Zakharov, V.A., Seltzer, V.B., Ivanov, A.V., Rostovtceva, J.A. \& Tarasova, L.O. 2004. The Upper Bajocian and Lower Bathonian in the section near Saratov. Vernadsky Museum Novitates 12, 1-39. [in Russian with English abstract]

Mitta, V., Glinskikh, L., Kostyleva, V., Dzyuba, O., Shurygin, B. \& Nikitenko, B. 2015. Biostratigraphy and sedimentary settings of the Bajocian-Bathonian beds of the Izhma River basin (European North of Russia). Neues Jahrbuch für Geologie und Paläontologie, Abhandlungen 277(3), 307-335. DOI 10.1127/njgpa/2015/0507

Mitta, V., Kostyleva, V., Dzyuba, O., Glinskikh, L., Shurygin, B., Setlzer, V., Ivanov, A. \& Urman, O. 2014. Biostratigraphy and sedimentary settings of the Upper Bajocian-Lower Bathonian of the vicinity of Saratov (Central Russia). Neues Jahrbuch für Geologie und Paläontologie, Abhandlungen 271(1), 95-121. DOI 10.1127/0077-7749/2014/0380 
MonTfort, P.D. de 1808. Conchyliologie systématique, et classification méthodique des coquilles; offrant leur figures, leur arrangement générique, leurs descriptions caractérisques, leurs noms, ainsi que leur synonymie en plusieurs langues. Coquilles univalves, cloisonnées, vol. I. 409 pp. Haussmann, Paris. DOI 10.5962/bhl.title.10571

MÜNSTER, G. GRAF ZU. 1839. Acanthoteuthis, ein neues Geschlecht der Cephalopoden zu der Familie der Loligineen oder Teuthidae (Owen) gehörend, 91-97. In Münster, G. Graf zu, Meyer, H.V. \& Wagner, R. (eds) Beiträge zur Petrefakten-Kunde, mit XVIII nach der Naturgezeichneten Tafeln, Heft 1. Buchner, Bayreth.

NAEF, A. 1922. Die fossilen Tintenfische; eine paläozoologische Monographie. 322 pp. G. Fischer, Jena. DOI 10.5962/bhl.title.2082

NAEF, A. 1926. Nomenklatorische Notiz betreffend die Gattung "Diploconus" Zittel, 1868. Pubblicazioni della Stazione Zoologica di Napoli 7, 383.

OgG, J.G., OGG, G.M. \& Gradstein, F.M. 2016. A Concise Geologic time Scale. 234 pp. Elsevier, Amsterdam, Boston \& Heidelberg.

Orbigny, A.D. D'. 1842-1851. Paléontologie française. Description zoologique et gdologique de tous les animaux mollusques et rayonnés fossiles de France, comprenant leur application à la reconnaissance des couches. Terrains oolithiques ou jurassiques. 1: Céphalopodes. 642 pp. Masson, Bertrand, d'Orbigny, Paris.

Orbigny, A.D. D'. 1850. Prodrôme de Paléonlologie stratigraphique universelle des animaux mollusques \& rayonnées faisant suite au cours élémentaire de paléontologie et de géologie stratigraphiques. Vol. 2.427 pp. Masson, Paris. DOI 10.5962/bhl.title.62810

PAGE, K.N. 1995. Biohorizons and zonules: intra-subzonal units in Jurassic ammonite stratigraphy. Palaeontology 38(4), 801-814.

Pearce, J.C. 1847. On the fossil Cephalopoda from the Oxford Clay constituting the genus Belemnoteuthis Pearce. Proceedings of the Geological Society of London 3, 592-594.

Price, G.D. \& Rogov, M.A. 2009. An isotopic appraisal of the Late Jurassic greenhouse phase in the Russian Platform. Palaeogeography, Palaeoclimatology, Palaeoecology 273(1-2), 41-49. DOI 10.1016/j.palaeo.2008.11.011

RIEGRAF, W. 1980. Revision der Belemniten des Schwäbischen Jura. Part 7. Palaeontographica A169(4-6), 128-206.

Riegraf, W. 1995. Cephalopoda dibranchiate fossiles (Coleoidea). Fossilium catalogus I, Animalia 133, 411 pp.

Riegraf, W., Janssen, N. \& Schmitt-Riegraf, C. 1998. Cephalopoda dibranchiate fossiles (Coleoidea). II. Supplementum ad mandibula fossiles ammonitorum et nautilorum (Rhyncholithi et Rhynchoteuthes, excl. aptychi et anaptychi). Fossilium catalogus I, Animalia 135, 519 pp.

Rogov, M.A. 2004. The Russian Platform as a key region for Volgian/Tithonian correlation: A review of the Mediterranean faunal elements and ammonite biostratigraphy of the Volgian stage. Rivista Italiana di Paleontologia e Stratigrafia 110(1), 321-328.
Rogov, M.A. 2010. A precise ammonite biostratigraphy through the Kimmeridgian-Volgian boundary beds in the Gorodischi section (Middle Volga area, Russia), and the base of the Volgian Stage in its type area. Volumina Jurassica VIII, 103-130.

Rogov, M. \& Bizikov, V. 2008. New data on Middle Jurassic-Lower Cretaceous Belemnotheutidae from Russia. What can shell tell about the animal and its mode of life. Acta Universitatis Carolinae, Geologica 49 (for 2006), 149-163.

Rogov, M. A., Gulyaev, D. B. \& Kiselev, D. N. 2012. Biohorizons as infrazonal biostratigraphic units: an attempt to refine the Jurassic stratigraphy based on ammonites. Stratigraphy and Geological Correlation 20(2), 211-229.

DOI 10.1134/S0869593812010066

Rogov, M.A., Wierzbowski, A. \& Shchepetova, E.V. 2017. Ammonite assemblages in the Lower to Upper Kimmeridgian boundary interval (Cymodoce to Mutabils zones) of Tatarstan (central European Russia) and their correlation importance. Neues Jahrbuch für Geologie und Paläontologie, Abhandlungen 285(2), 161-185.

DOI 10.1127/njgpa/2017/0675

SACHS, V.N. \& NALnJAEVA, T.I. 1966. The Upper Jurassic and Lower Cretaceous belemnites of the north of the USSR. Genera Pachyteuthis and Acroteuthis. 260 pp. Nauka, Moscow-Leningrad. [in Russian]

Sachs, V.N. \& NalnjaeVA, T.I. 1967. Recognition of the superfamily Passaloteuthaceae in the suborder Belemnoidea (Cephalopoda, Dibranchia, Decapoda). Doklady Akademii Nauk SSSR 173(2), 438-441. [in Russian]

SACHS, V.N. \& NALnJaEVA, T.I. 1975. The Early and Middle Jurassic belemnites of the north of the USSR: Megateuthinae and Pseudodicoelitinae. Academy of Sciences of the USSR, Siberian branch, Transactions of the Institute of Geology and Geophysics 239, 1-192. [in Russian]

Schlegelmilch, R. 1998. Die Belemniten des Süddeutschen Jura. 151 pp. Gustav Fischer, Stuttgart, Jena, Lübeck, Ulm.

DOI 10.1007/978-3-8274-3083-0

SCOTESE, C.R. 2016. PALEOMAP PaleoAtlas for GPlates and the PaleoData Plotter Program. Updated at:

https://www.earthbyte.org/paleomap-paleoatlas-for-gplates/, accessed 01 November, 2016.

Spath, L.F. 1927. Revision of the Jurassic Cephalopod fauna of Kachh (Cutch). Part 1. Palaeontologia indica, New series 9/2, 279-350.

Spath, L.F. 1939. Sôbre um novo belemnitóide (Conoteuthis renniei) de Aptiano de Moçambique/ On a new belemnoid (Conoteuthis renniei) from the Aptian of the Colony of Moçambique. Serviços de Industria, Minas e Geologia. Série de Geologia e Minas. Memórias e Comunicações. Boletim 2, 5-16.

Stolley, E. 1919. Die Systematik der Belemniten. Jahresbericht des Niedersächsischen Geologischen Vereins XI (for 1918), $1-59$.

Stoyanova-Vergilova, M.P. 1983. Genus Paramegateuthis Gustomesov (Belemnitida) in Bulgaria. Annuaire de l'Université de Sofia "Kliment Ohridski", Faculté de Géologie et Géographie 73(1), 251-259. 
Vischiakoff, N. 1875. Sur les Aptychus de Gorodisché. Bulletin de la Société impériale des naturalistes de Moscou 49(3), 175-178.

Weis, R. \& Mariotti, N. 2008. A belemnite fauna from the Aalenian-Bajocian boundary beds of the Grand Duchy of Luxembourg (NE Paris Basin). Bollettino della Società Paleontologica Italiana 46(2-3), 149-174.

WeIs, R., SADKI, D. \& MARIOTTI, N. 2017. Aalenian-Bajocian belemnites from the Middle and High Atlas, Morocco: taxonomy, biostratigraphy and palaeobiogeographical affinities. Neues Jahrbuch für Geologie und Paläontologie, Abhandlungen 284(2), 215-240. DOI 10.1127/njgpa/2017/0659

Wierzbowski, H., Rogov, M.A., Matyja, B.A., Kiselev, D. \& Ippolitov, A. 2013. Middle-Upper Jurassic (Upper Callovian-Lower Kimmeridgian) stable isotope and elemental records of the Russian Platform: indices of oceanographic and climatic changes. Global and Planetary Change 107, 196-212. DOI 10.1016/j.gloplacha.2013.05.011

Zakharov, V.A., Baudin, F., Dzyuba, O.S., Daux, V., Zverev,
K.V. \& RENARD, M. 2005. Isotopic and faunal record of high paleotemperatures in the Kimmeridgian of Subpolar Urals. Russian Geology and Geophysics 46(1), 1-19.

Zakharov, V.A., Rogov, M.A., Dzyuba, O.S., ŽÁK, K., KošŤÁÁ, M., Pruner, P., Skupien, P., Chadima, M., Mazuch, M. \& Nikitenko, B.L. 2014. Palaeoenvironments and palaeoceanography changes across the Jurassic/Cretaceous boundary in the Arctic realm: case study of the Nordvik section (north Siberia, Russia). Polar Research 33, 19714. DOI 10.3402/polar.v33.19714

ZitTel, K.A. von 1868. Diploconus, ein neues Genus aus der Familie der Belemniten. Neues Jahrbuch für Mineralogie, Geologie und Paläontologie 1868, 548-552.

Zittel, K.A. von 1881-1885. Handbuch der Palaeontologie. 1 Abt. Palaeozoologie. Bd. 2. Mollusca und Arthropoda. 893 pp. Oldenbourg, München, Leipzig.

ZitTel, K.A. vON 1895. Grundzüge der Paläontologie (Paläzoologie). 971 pp. Oldenbourg, München, Leipzig. 